\title{
Continuous Hecke algebras
}

\author{
Pavel Etingof, Wee Liang Gan, Victor Ginzburg \\ Table of Contents \\ 1. Introduction \\ 2. Continuous Hecke algebras \\ 3. Continuous symplectic reflection algebras and Cherednik algebras \\ 4. Infinitesimal Hecke algebras \\ 5. Representation theory of continuous Cherednik algebras \\ 6. Case of wreath-products
}

The theory of $P B W$ properties of quadratic algebras, to which this paper aims to be a modest contribution, originates from the pioneering work of Drinfeld (see [Dr1]). In particular, as we learned after publication of [EG] (to the embarrassment of two of us!), symplectic reflection algebras, as well as $\mathrm{PBW}$ theorems for them, were discovered by Drinfeld in the classical paper [Dr2] 15 years before [EG] (namely, they are a special case of degenerate affine Hecke algebras for a finite group $G$ introduced in [Dr2], Section 4).

It is our great pleasure to dedicate this paper to Vladimir Drinfeld on the occasion of his 50-th Birthday.

\section{Introduction}

In Dr2, Section 4, Drinfeld introduced the family of degenerate affine (=graded) Hecke algebras attached to any finite group $G$ and its linear finite dimensional complex representation $V$. The goal of this paper is to define and study the "continuous" generalization of these algebras, in which the group $G$ is a reductive algebraic group, and $V$ is its algebraic representation. We call this generalization continuous Hecke algebras. They include continuous symplectic reflection algebras and continuous Cherednik algebras, which are generalizations of symplectic reflection algebras from [EG] and rational Cherednik algebras.

A motivation for studying continuous Hecke algebras comes from the fact that their representation theory (which is yet to be developed) unifies the representation theories of real reductive groups, Drinfeld-Lusztig degenerate affine Hecke algebras, and symplectic reflection algebras of [EG] (in particular, rational Cherednik algebras). 
Let us briefly describe the contents of the paper. Let $G$ be a reductive algebraic group ${ }^{1}$ with an algebraic representation $\rho: G \rightarrow G L(V)$. Let $\mathcal{O}(G)^{*}$ be the algebra, with respect to convolution, of algebraic distributions on $G$ (see subsection 2.1). This algebra has a natural $\mathcal{O}(G)$-module structure.

Let $\kappa \in\left(\mathcal{O}(G)^{*} \otimes \wedge^{2} V^{*}\right)^{G}$. Following the idea of [Dr2], we define the algebra $\mathrm{H}_{\kappa}$ to be the quotient of $T V \rtimes \mathcal{O}(G)^{*}$ by the "main commutation relation"

$$
[x, y]=\kappa(x, y), \quad x, y \in V,
$$

and we are interested in finding those $\kappa$ for which $\mathrm{H}_{\kappa}$ is a flat deformation of $\mathrm{H}_{0}=$ $S V \rtimes \mathcal{O}(G)^{*}$ (the PBW property). As in [EG], it turns out that flatness is equivalent to the Jacobi identity

$$
\kappa(x, y)\left(z-z^{g}\right)+\kappa(y, z)\left(x-x^{g}\right)+\kappa(z, x)\left(y-y^{g}\right)=0, x, y, z \in V, g \in G .
$$

We call the algebras $\mathrm{H}_{\kappa}$ satisfying the PBW property (equivalently, the Jacobi identity) continuous Hecke algebras.

One of the main problems treated in this paper is to classify continuous Hecke algebras, i.e. to find explicitly all distributions $\kappa$ satisfying the Jacobi identity. In the case when $G$ is finite, the answer is given in Dr2 (see also [RS]). However, for an infinite $G$ the situation is more complicated and we don't have a complete answer; so we present some partial results.

Let $\Phi \subset G$ be the closed subscheme of $G$ defined by the equation $\wedge^{3}\left(1-\left.g\right|_{V}\right)=0$. Our first result is that if $\kappa$ satisfies the Jacobi identity then it is supported on $\Phi$.

Next, let $\theta \in\left(\mathcal{O}(\Phi)^{*} \otimes \wedge^{2} V^{*}\right)^{G}$, and $\tau \in\left(\mathcal{O}(\operatorname{Ker} \rho)^{*} \otimes \wedge^{2} V^{*}\right)^{G}$. We show that the distribution

$$
\kappa(x, y):=\tau(x, y)+\theta((1-g) x,(1-g) y)
$$

satisfies the Jacobi identity and hence defines a continuous Hecke algebra. This is the general answer if the group $G$ is finite.

Another collection of examples comes from symmetric pairs $(\mathbf{G}, \mathbf{K})$ of reductive groups. In this case $\mathbf{H}_{\kappa}=A(\mathbf{G}, \mathbf{K})$, the algebra of distributions on $\mathbf{G}$ set-theoretically supported on $\mathbf{K}$.

Further, we consider the special case when the space $V$ carries a $G$-invariant symplectic form $\omega$. In this case, let $\Sigma$ be the closed subscheme of $G$ defined by the equation $p \circ \wedge^{3}\left(1-\left.g\right|_{V}\right)=0$, where $p: \wedge^{3} V \rightarrow V$ is the projection defined by $\omega$. Let

$$
\kappa(x, y)=\omega(x, y) t+\omega((1-g) x,(1-g) y) c,
$$

where $t$ is a $G$-invariant distribution on $\operatorname{Ker} \rho$, and $c$ is a $G$-invariant distribution on $\Sigma$. Here $\omega((1-g) x,(1-g) y) c$ demotes the product of the regular function $\omega((1-g) x,(1-g) y)$ by the algebraic distribution $c$, using the $\mathcal{O}(G)$-module structure on $\mathcal{O}(G)^{*}$.

\footnotetext{
${ }^{1}$ Unless otherwise specified, all algebraic groups and Lie algebras in this paper are over $\mathbb{C}$.
} 
We show that $\kappa$ satisfies Jacobi identity, and call the corresponding algebras $\mathrm{H}_{\kappa}$ continuous symplectic reflection algebras. If $G$ is finite, and $\rho$ is a faithful representation, they coincide with symplectic reflection algebras defined in [EG].

Now let us assume in addition that $V=\mathfrak{h} \oplus \mathfrak{h}^{*}$, where $\mathfrak{h}$ is a representation of $G$ (and the form $\omega$ is given by the natural pairing between $\mathfrak{h}$ and $\left.\mathfrak{h}^{*}\right)$. In this case we show that $\kappa$ satisfies Jacobi identity if $\kappa(y, x)=0$ if $y, x \in \mathfrak{h}$ or $y, x \in \mathfrak{h}^{*}$, and

$$
\kappa(y, x)=(y, x) t+(y,(1-g) x) c, y \in \mathfrak{h}, x \in \mathfrak{h}^{*} .
$$

where $t$ is a $G$-invariant distribution on $\operatorname{Ker} \rho$, and $c$ is an invariant distribution on the closed subscheme $\Psi \subset G$, "of complex reflections", defined by the equation $\wedge^{2}\left(1-\left.g\right|_{\mathfrak{h}}\right)=0$. We call algebras $\mathrm{H}_{\kappa}$ for such $\kappa$ continuous Cherednik algebras. If $G$ is finite and $\rho$ is a faithful representation, they coincide with the rational Cherednik algebras considered in EG.

One of our main results is that if $\mathfrak{h}$ is irreducible of real or complex type and faithful then the classes of continuous Hecke algebras, continuous symplectic reflection algebras, and continuous Cherednik algebras are the same.

We consider in detail the examples $G=G L_{n}, O_{n}, S p_{2 n}$, and $V=\mathbb{C}^{2 n}$ with the natural symplectic form. ${ }^{2}$ In all these examples, any continuous Hecke algebra $\mathrm{H}_{\kappa}$ is a continuous symplectic reflection algebra, and we classify explicitly the possible distributions $c$. In particular, for $G=O_{n}$ we find that there is just one essential parameter, while for $G=G L_{n}$ and $G=S p_{2 n}$ there are infinitely many.

If the distribution $\kappa$ is set-theoretically supported at 1 (i.e. belongs to $\mathcal{U} \mathfrak{g}$, where $\mathfrak{g}$ is the Lie algebra of $G$ ), then one can define the algebra $\mathscr{H}_{\kappa}$ to be the quotient of $T V \rtimes \mathcal{U} \mathfrak{g}$ by the main commutation relation. It is a flat deformation of $S V \rtimes \mathcal{U} \mathfrak{g}$ if and only if $\kappa$ satisfies the Jacobi identity; in this case we call $\mathrm{H}_{\kappa}$ an infinitesimal Hecke algebra. We write such $\kappa$ explicitly in the cases $G=G L_{n}, G=S p_{2 n}$ (in the case $G=O_{n}$, there are no interesting algebras $\mathscr{H}_{\kappa}$ ).

An interesting problem is to develop representation theory of the algebras $\mathrm{H}_{\kappa}$ and $\mathscr{H}_{\kappa}$. In the case of $G=S L_{2}, V=\mathbb{C}^{2}$, this was done in $[\mathrm{Kh}$, but other examples (apart from finite $G$ ) remain mostly unexplored. We begin to tackle this problem by introducing the category $\mathcal{O}$ and studying the Dunkl-Cherednik representation of continuous Cherednik algebras.

Remark 1.1. Note that continuous representations of the algebra $A(\mathbf{G}, \mathbf{K})$ are nothing but Harish-Chandra modules for the pair $(\mathbf{G}, \mathbf{K})$. Thus, the representation theory of continuous Hecke algebras is a generalization of the representation theory of real reductive groups.

\footnotetext{
${ }^{2}$ The example $G=S p_{2}=S L_{2}$ was considered earlier in [Kh].
} 
At the end of the paper, we consider the case of wreath products $G=S_{n} \ltimes \Gamma^{n}$, where $\Gamma$ is a reductive subgroup of $S L_{2}$, and $V=\mathbb{C}^{2 n}$. We consider in detail the three cases in which $\Gamma$ is infinite, and generalize the results of Montarani on the classification of representations of $\mathrm{H}_{\kappa}$ irreducible over $G$ (proved in [M] in the case when $\Gamma$ is finite).

Acknowledgments. The work of P.E. was partially supported by the NSF grant DMS-9988796. The work of V.G. was partially supported by the NSF grant DMS-0303465. The work of W.L.G. was partially supported by the NSF grant DMS-0401509. The work of P.E. and V.G. was partially supported by the CRDF grant RM1-2545-MO-03.

\section{Continuous Hecke algebras}

2.1 Algebraic distributions. Let $X$ be an affine scheme of finite type over $\mathbb{C}$. We shall denote by $\mathcal{O}(X)$ the algebra of regular functions on $X$. An algebraic distribution on $X$ is an element in the dual space $\mathcal{O}(X)^{*}$ of $\mathcal{O}(X)$. For $c \in O(X)^{*}$ and $f \in \mathcal{O}(X)$, we will denote the value $c(f)$ by $(c, f)$.

The space $\mathcal{O}(X)^{*}$ is naturally equipped with the weak (inverse limit) topology. Note also that $\mathcal{O}(X)^{*}$ is a module over $\mathcal{O}(X)$ : for any $f \in \mathcal{O}(X)$ and $\mu \in \mathcal{O}(X)^{*}$ we can define the element $f \mu=\mu f \in \mathcal{O}(X)^{*}$ by $(f \mu, g)=(\mu, f g)$.

Let $Z$ be a closed subscheme of $X$, and write $I(Z)$ for its defining ideal in $\mathcal{O}(X)$. We say that an algebraic distribution $\mu$ on $X$ is supported on the scheme $Z$ if $\mu$ annihilates $I(Z)$. Clearly, the space of algebraic distributions on $X$ supported on $Z$ is naturally isomorphic to the space of algebraic distributions on $Z$.

Now assume that $Z$ is reduced. We say that $\mu \in \mathcal{O}(X)^{*}$ is scheme-theoretically (respectively, set-theoretically) supported on the set $Z$ if $\mu$ annihilates $I(Z)$ (respectively, some power of $I(Z))$.

Example 2.1. For each point $a \in X$, the delta function $\delta_{a} \in \mathcal{O}(X)^{*}$ is defined by $\delta_{a}(f):=f(a)$ where $f \in \mathcal{O}(X)$. It is scheme-theoretically supported at the point $a$, and its derivatives are set-theoretically supported at this point.

Let $G$ be a reductive algebraic group. Since $\mathcal{O}(G)$ is a coalgebra, its dual space $\mathcal{O}(G)^{*}$ is an algebra under convolution. The unit of this algebra is the delta function $\delta_{1}$ of the identity element $1 \in G$.

Note that a continuous representation of the algebra $\mathcal{O}(G)^{*}$ is the same thing as a locally finite $G$-module (i.e. a $G$-module which is a direct sum of finite dimensional algebraic representations of $G$ ).

Suppose that $G$ acts on $X$. Then $G$ acts also on $\mathcal{O}(X)$ and $\mathcal{O}(X)^{*}$. We have $\mathcal{O}(X)=\bigoplus_{V} M_{V} \otimes V$, where $V$ runs over the irreducible representations of $G$, and $M_{V}$ are multiplicity spaces. Thus, $\mathcal{O}(X)^{*}=\prod_{V} M_{V}^{*} \otimes V^{*}$. In particular, $\mathcal{O}(G)^{*}=\prod_{V} V \otimes V^{*}$ as a $G \times G$-module. 
Recall that the categorical quotient $X / G$ is an affine scheme of finite type with $\mathcal{O}(X / G)=$ $\mathcal{O}(X)^{G}$. It is clear that the space $\left(\mathcal{O}(X)^{*}\right)^{G}$ of invariant algebraic distributions on $X$ is naturally isomorphic to the space $\mathcal{O}(X / G)^{*}$ of algebraic distributions on the quotient $X / G$. We will denote this space by $\mathrm{C}(X)$. Clearly, if $Z \subset X$ is a $G$-invariant closed subscheme, then $\mathrm{C}(Z)$ is naturally a subspace of $\mathrm{C}(X)$.

2.2 Definition and properties of continuous Hecke algebras. Let $G$ be a reductive algebraic group (not necessarily connected), and $\rho: G \rightarrow G L(V)$ be a finite dimensional algebraic representation of $G$. The semi-direct product $T V \rtimes \mathcal{O}(G)^{*}$ is the algebra generated by $x \in V$ and $\mu \in \mathcal{O}(G)^{*}$ with the relations

$$
\mu \cdot x=\sum_{i} v_{i} \cdot\left(v_{i}^{*}, g x\right) \mu \quad \text { for all } x \in V, \mu \in \mathcal{O}(G)^{*},
$$

where $v_{i}$ is a basis of $V$ and $v_{i}^{*}$ the dual basis of $V^{*}$. (Here $\left(v_{i}^{*}, g x\right) \mu$ denotes the product of the regular function $\left(v_{i}^{*}, g x\right)$ by the distribution $\left.\mu\right)$. Given a skew-symmetric $G$-equivariant $\mathbb{C}$-bilinear pairing $\kappa: V \times V \rightarrow \mathcal{O}(G)^{*}$ (where $G$ acts on $\mathcal{O}(G)^{*}$ by conjugation), let $\mathrm{H}_{\kappa}=\mathrm{H}_{\kappa}(G)$ be the quotient of $T V \rtimes \mathcal{O}(G)^{*}$ by the relations

$$
[x, y]=\kappa(x, y) \quad \text { for all } x, y \in V .
$$

Define a filtration on $\mathrm{H}_{\kappa}$ by setting $\operatorname{deg}(x)=1, \operatorname{deg}(\mu)=0$ for $x \in V, \mu \in \mathcal{O}(G)^{*}$. Note that $\mathrm{H}_{0}=S V \rtimes \mathcal{O}(G)^{*}$. There is a natural map $\mathrm{H}_{0} \rightarrow \operatorname{gr}\left(\mathrm{H}_{\kappa}\right)$ which is a surjective graded algebra homomorphism. We say that the $P B W$ property holds for $\mathrm{H}_{\kappa}$ if this morphism is an isomorphism.

For $x \in V$, let $x^{g}$ denote the result of the action of $g$ on $x$.

Theorem 2.4. The algebra $\mathrm{H}_{\kappa}$ has the $P B W$ property if and only if $\kappa$ satisfies the Jacobi identity

$$
\left(z-z^{g}\right) \kappa(x, y)+\left(y-y^{g}\right) \kappa(z, x)+\left(x-x^{g}\right) \kappa(y, z)=0 \quad \text { for all } x, y, z \in V .
$$

Proof. The algebra $\mathrm{H}_{0}$ is Koszul. Therefore, by the results of [BG], BGS] (see also [EG], $\S 2])$, the PBW property is equivalent to the Jacobi identity ${ }^{3}$

$$
[\kappa(x, y), z]+[\kappa(z, x), y]+[\kappa(y, z), x]=0 \quad \text { for all } x, y, z \in V .
$$

By (2.2), we have

$$
[z, \kappa(x, y)]=\left(z-z^{g}\right) \kappa(x, y) \in(V \otimes \mathcal{O}(G)) \otimes_{\mathcal{O}(G)} \mathcal{O}(G)^{*}=V \otimes \mathcal{O}(G)^{*} .
$$

Hence, the Jacobi identity (2.6) is equivalent to equation (2.5), as needed.

\footnotetext{
${ }^{3}$ Strictly speaking, the result of [BGS] is stated in the situation when the degree zero part of the algebra is finite dimensional semisimple; however, one can check that all arguments extend to the case when the degree zero part is the inverse limit of finite dimensional semisimple algebras, in particular when the degree zero part is $\mathcal{O}(G)^{*}$.
} 
Definition 2.7. If $\mathrm{H}_{\kappa}$ has the PBW property (equivalently, $\kappa$ satisfies the Jacobi identity) then $\mathrm{H}_{\kappa}$ is called a continuous Hecke algebra.

Let $\Phi \subset G$ be the closed subscheme defined by the equation $\wedge^{3}\left(1-\left.g\right|_{V}\right)=0$. Then the set of closed points of $\Phi$ is the set $S$ of elements of $G$ such that the rank of the operator $1-g: V \rightarrow V$ is $\leq 2$.

Proposition 2.8. If the $P B W$ property holds for $\mathrm{H}_{\kappa}$, then $\kappa(x, y)$ is supported on the scheme $\Phi$ for all $x, y \in V$. (In particular, $\kappa(x, y)$ is set-theoretically supported on $S$.

Proof. Here and below, we will use the following notation: for any $v_{1}, \ldots, v_{k}, x, y \in V$, we write

$\left(v_{1}, \ldots, v_{k} \mid x, y\right)$ for $\left(v_{1}-v_{1}^{g}\right) \wedge \cdots \wedge\left(v_{k}-v_{k}^{g}\right) \kappa(x, y) \in\left(\wedge^{k} V \otimes \mathcal{O}(G)\right) \otimes \otimes_{\mathcal{O}(G)} \mathcal{O}(G)^{*}=\wedge^{k} V \otimes \mathcal{O}(G)^{*}$.

Thus, we can rewrite the condition (2.5) as

$$
(z \mid x, y)+(x \mid y, z)+(y \mid z, x)=0 \quad \text { for all } x, y, z \in V .
$$

To prove the proposition, we will need two lemmas.

Lemma 2.10. If (2.9) holds, then $(z, u \mid x, y)=(x, y \mid z, u)$ for all $x, y, z, u \in V$.

Proof. We have

$$
\begin{aligned}
& (u, z \mid x, y)=-(u, x \mid y, z)-(u, y \mid z, x), \\
& (z, u \mid x, y)=-(z, x \mid y, u)-(z, y \mid u, x) .
\end{aligned}
$$

Subtracting the first equation from the second equation, and using (2.9), we get

$$
\begin{aligned}
2(z, u \mid x, y) & =((x, z \mid y, u)+(x, u \mid z, y))+((y, z \mid u, x)+(y, u \mid x, z)) \\
& =(x, y \mid z, u)+(y, x \mid u, z) \\
& =2(x, y \mid z, u) .
\end{aligned}
$$

Lemma 2.11. If (2.9) holds, then $(z, u, v \mid x, y)=0$ for all $x, y, z, u, v \in V$.

Proof. By Lemma 2.10, we have

$$
\begin{aligned}
& (v, z, u \mid x, y)=(v, x, y \mid z, u), \\
& (u, v, z \mid x, y)=(u, x, y \mid v, z), \\
& (z, u, v \mid x, y)=(z, x, y \mid u, v) .
\end{aligned}
$$

Adding these three equations, we get

$$
3(z, u, v \mid x, y)=(x, y, v \mid z, u)+(x, y, u \mid v, z)+(x, y, z \mid u, v)=0 .
$$


Now we prove Proposition 2.8. The PBW property is equivalent to condition (2.9). Hence, the proposition is immediate from Lemma 2.11.

2.3 Examples of continuous Hecke algebras. Let $\Theta:=\left(\mathcal{O}(\Phi)^{*} \otimes \wedge^{2} V^{*}\right)^{G}$, and $\Theta_{0} \subset \Theta$ be the subspace of those $\theta \in \Theta$ for which $\theta((1-g) x,(1-g) y)=0$ for any $x, y \in V$. Let $\theta \in \Theta / \Theta_{0}$, and let $\tau \in\left(\mathcal{O}(\operatorname{Ker} \rho)^{*} \otimes \wedge^{2} V^{*}\right)^{G}$. Define the distribution $\kappa$ by

$$
\kappa(x, y)=\tau(x, y)+\theta((1-g) x,(1-g) y) .
$$

Theorem 2.13. The algebra $\mathrm{H}_{\kappa}$ for such $\kappa$ has the PBW property.

Proof. Since $\left(u-u^{g}\right) \tau=0$, the Jacobi identity reduces to the relation

$$
\left(z-z^{g}\right) \theta\left(x-x^{g}, y-y^{g}\right)+\left(y-y^{g}\right) \theta\left(z-z^{g}, x-x^{g}\right)+\left(x-x^{g}\right) \theta\left(y-y^{g}, z-z^{g}\right)=0 .
$$

This relation holds since $\theta$ is alternating and is supported on $\Phi$. We are done.

Let us analyze in more detail the special case of finite group $G$ (this analysis is given in [Dr2] and in a more expanded form in [RS] ). In this case, if $\kappa$ satisfies the Jacobi identity, then by Proposition 2.8, $\kappa$ is supported on the set $S$. Therefore, the main commutation relation of the algebra $\mathrm{H}_{\kappa}$ takes the form

$$
[x, y]=\tau(x, y)+\sum_{g \in S, \rho(g) \neq 1} \theta_{g}(x, y) g
$$

where $\tau \in\left(\mathbb{C}[\operatorname{Ker} \rho] \otimes \wedge^{2} V^{*}\right)^{G}$, and $\theta_{g}$ is a 2-form on $V$. It follows from the Jacobi identity that $\theta_{g}(x, y)=0$ if $x, y \in \operatorname{Ker}\left(1-\left.g\right|_{V}\right)$. Since $\theta_{g}$ is $g$-invariant, this implies that the kernel of $\theta_{g}$ contains $\operatorname{Ker}\left(1-\left.g\right|_{V}\right)$, and hence $\theta_{g}$ is uniquely determined by $g$ up to scaling. We also see that $\kappa(x, y)$ is given by formula (2.12) for an appropriate $\theta$.

Furthermore, to secure the $G$-invariance of the right hand side of the commutation relation, we need to require that the family of forms $\theta_{g}$ is $G$-invariant. If $C \subset S$ is a conjugacy class, then a nonzero invariant family $\left\{\theta_{g}, g \in C\right\}$ is unique up to scaling if exists, and it exists if and only if the rank of $1-\left.g\right|_{V}$ is exactly 2 , and the centralizer $Z_{g}$ of $g \in C$ acts trivially on the 1 -dimensional space $\wedge^{2} \operatorname{Im}(1-g)$. Let us call such conjugacy class admissible, and denote the set of admissible conjugacy classes by $S_{\text {adm. }}$. Thus we have

$$
[x, y]=\tau(x, y)+\sum_{g \in S_{\text {adm }}} \theta_{g}(x, y) g .
$$

Note that since $g \in Z_{g}$, we find that if $g$ is admissible then $\operatorname{det}(g)=1$, so $g$ is conjugate in $G L(V)$ to $\operatorname{diag}\left(\lambda, \lambda^{-1}, 1, \ldots, 1\right)$. This shows that the study of degenerate Hecke algebras $\mathrm{H}_{\kappa}$ essentially reduces to the case when $G \subset S L(V)$.

Remark 2.14. It is explained in Dr2, RS that the class of algebras $\mathrm{H}_{\kappa}$ with $\mathrm{PBW}$ property for finite $G$ in particular contains degenerate affine Hecke algebras for finite Coxeter groups, introduced by Drinfeld and Lusztig. 
2.4 Continuous Hecke algebras and symmetric pairs. Let $(\mathbf{G}, \mathbf{K})$ be a symmetric pair of complex reductive groups. Let $\mathfrak{g}, \mathfrak{k}$ be the Lie algebras of $\mathbf{G}, \mathbf{K}$. Then we have the standard decomposition $\mathfrak{g}=\mathfrak{k} \oplus \mathfrak{p}$. Let $A(\mathbf{G}, \mathbf{K})$ be the algebra of algebraic distributions on $\mathbf{G}$ set-theoretically supported on $\mathbf{K}$. It is generated by $\mathcal{O}(\mathbf{K})^{*}$ and $\mathfrak{p}$, with appropriate relations.

It is clear that $A(\mathbf{G}, \mathbf{K})$ is isomorphic to $\mathrm{H}_{\kappa}(\mathbf{K})$ for a particular distribution $\kappa$, given by $\kappa(x, y)=[x, y] \in \mathfrak{k} \subset \mathcal{O}(\mathbf{K})^{*}$, where $x, y \in \mathfrak{p}$ (here we regard elements of $\mathfrak{k}$ as distributions on $\mathbf{K}$ supported in the first order neighborhood of the identity). This means that $A(\mathbf{G}, \mathbf{K})$ is a continuous Hecke alegbra.

In fact, one also has the converse result.

Theorem 2.15. Assume that $\mathbf{K} \subset G L(E)$, and $\kappa: \wedge^{2} E \rightarrow \mathfrak{k} \subset \mathcal{O}(\mathbf{K})^{*}$ is an invariant map, such that $\mathrm{Ker} \kappa=0$, and the algebra $\mathrm{H}_{\kappa}(\mathbf{K})$ satisfies the $P B W$ property. Then $\mathrm{H}_{\kappa}(\mathbf{K})$ comes from a symmetric pair, as above. Moreover, the group $\mathbf{G}$ is semisimple.

The proof of the theorem is based on the following lemma. ${ }^{4}$

Let $\mathbf{K}$ be a reductive algebraic group over $\mathbb{C}$ with Lie algebra $\mathfrak{k}$, and $E$ a faithful algebraic representation of $\mathbf{K}$. Let $\kappa: \wedge^{2} E \rightarrow \mathfrak{k}$ be a homomorphism of $\mathbf{K}$-modules, such that $\operatorname{Ker} \kappa=0$. Let $\mathfrak{g}=\mathfrak{k} \oplus E$. We have a natural linear map [,] : $\wedge^{2} \mathfrak{g} \rightarrow \mathfrak{g}$, which extends the Lie bracket on $\mathfrak{k}$ and the natural $\mathfrak{k}$-actions on $E$, and satisfies the condition: $\left.[]\right|_{,E \otimes E}=\kappa$.

Lemma 2.16. Suppose that [,] is a Lie bracket on $\mathfrak{g}$. Then $\mathfrak{g}$ is a semisimple Lie algebra.

Proof. Let $B_{\mathfrak{g}}, B_{\mathfrak{k}}$ denote the Killing forms of the corresponding Lie algebras. We have $B_{\mathfrak{g}}(a, b)=B_{\mathfrak{k}}(a, b)+\operatorname{Tr}_{E}(a b)$ for $a, b \in \mathfrak{k}$. Since $E$ is faithful, for any $a \neq 0$ in the Lie algebra $\mathfrak{k}_{\mathbb{R}}$ of a compact form $\mathbf{K}_{\mathbb{R}}$ of $\mathbf{K}$, we have $B_{\mathfrak{g}}(a, a)<0$. Hence the kernel of $B_{\mathfrak{g}}$ is contained in $E$. This kernel is an ideal in $\mathfrak{g}$, so it is contained in the kernel of $\kappa$. Hence it must be zero, so $B_{\mathfrak{g}}$ is nondegenerate and $\mathfrak{g}$ is semisimple.

Proof. (of Theorem 2.15). Let $\mathfrak{g}=\mathfrak{k} \oplus E$. Since $\mathrm{H}_{\kappa}$ satisfies PBW property, we have $\mathfrak{g} \subset \mathrm{H}_{\kappa}(\mathbf{K})$. Then $\mathfrak{g}$ is closed under bracket, so it is a Lie algebra as in Lemma 2.16. It follows from Lemma 2.16 that $\mathfrak{g}$ is semisimple. We are done.

\section{Continuous symplectic reflection algebras and Cherednik algebras}

In this section we will consider the special case when the representation $V$ has a $G$-invariant symplectic form $\omega$.

\footnotetext{
${ }^{4}$ The first author is grateful to D. Vogan for explaining to him a proof of this lemma.
} 
3.1 Continuous symplectic reflection algebras. Let $\Sigma \supset \Phi$ be the closed subscheme of $G$ defined by the equation $p \circ \wedge^{3}\left(1-\left.g\right|_{V}\right)=0$, where $p: \wedge^{3} V \rightarrow V$ is the projection defined by contracting the first two components using $\omega$.

Theorem 3.1. Let $t \in\left(\mathcal{O}(\operatorname{Ker} \rho)^{*}\right)^{G}$ and $c \in \mathrm{C}(\Sigma)$. If

$$
\kappa(x, y)=\omega(x, y) t+\omega((1-g) x,(1-g) y) c \quad \text { for all } x, y \in V,
$$

then the $P B W$ property holds for $\mathrm{H}_{\kappa}$.

Proof. We shall show that (2.5) holds when $\kappa$ is of the form (3.2). First note that $\left(v-v^{g}\right) t=$ 0 . Hence, we have to show that $f(x, y, z ; g) c=0$, where

$f(x, y, z ; g):=\left(z-z^{g}\right) \omega\left(x-x^{g}, y-y^{g}\right)+\left(x-x^{g}\right) \omega\left(y-y^{g}, z-z^{g}\right)+\left(y-y^{g}\right) \omega\left(z-z^{g}, x-x^{g}\right)$.

This is clear, since $c$ is supported on $\Sigma$.

Let $S$ be the set of symplectic reflections in $G$, i.e. elements $s \in G$ such that the rank of the operator $1-s$ on $V$ is $\leq 2$. It is easy to see that the set of closed points of $\Sigma$ is $S \cup Q$, where $Q$ is the subvariety of elements $g \in G$ such that $(1-g)^{2}=0$. In particular, any semisimple element $g \in \Sigma(\mathbb{C})$ belongs to $S$, and if $\lambda \neq 1$ is an eigenvalue of $g$, then $\omega((1-g) x,(1-g) y)=(1-\lambda)\left(1-\lambda^{-1}\right) \omega_{g}(x, y)$, where $\omega_{g}(x, y)=\omega\left(x^{\prime}, y^{\prime}\right), x^{\prime}, y^{\prime}$ being the projections of $x, y$ on the image of $1-g$ along the kernel of $1-g$. Hence, if $G$ is a finite group and $V$ is a faithful representation then the algebra of Theorem 3.1 is exactly the symplectic reflection algebra of [EG].

Thus, algebras $\mathrm{H}_{\kappa}$ with $\kappa$ being of the form (3.2) are natural continuous analogs of symplectic reflection algebras of [EG]. We will therefore call $\mathrm{H}_{\kappa}$ a continuous symplectic reflection algebra.

3.2 Continuous Cherednik algebras. Consider separately the special case $V=\mathfrak{h} \oplus \mathfrak{h}^{*}$, $G \subset G L(\mathfrak{h}) \subset S p(V)$, where $\mathfrak{h}$ is a complex vector space, and the form $\omega$ is the pairing between $\mathfrak{h}$ and $\mathfrak{h}^{*}$. In this case, let $\Psi$ be the closed subscheme of $G$ defined by the equation $\wedge^{2}\left(1-\left.g\right|_{\mathfrak{h}}\right)=0$. Obviously, $\Psi \subset \Phi$, and the set of closed points of $\Psi$ is the set $S$ of all complex (=symplectic) reflections in $G$, i.e., $s \in G$ such that the rank of $1-\left.s\right|_{\mathfrak{h}}$ is $\leq 1$.

Theorem 3.3. Let $t \in\left(\mathcal{O}(\operatorname{Ker} \rho)^{*}\right)^{G}$ and $c \in \mathrm{C}(\Psi)$. If $\kappa(x, y)=0$ for $x, y \in \mathfrak{h}$ or $x, y \in \mathfrak{h}^{*}$, and one has

$$
\kappa(x, y)=(y, x) t+(y,(1-g) x) c \quad \text { for all } x \in \mathfrak{h}^{*}, y \in \mathfrak{h}
$$

then the PBW property holds for $\mathrm{H}_{\kappa}$. 
Proof. We shall show that (2.5) holds when $\kappa$ is of the form (3.4). Similarly to the proof of Theorem 3.1] we have to show that $h_{1}(x, y, z ; g) c=0, h_{2}(x, y, u ; g) c=0$, where

$$
\begin{aligned}
& h_{1}(x, y, z ; g):=\left(z-z^{g}\right)\left(x, y-y^{g}\right)-\left(x-x^{g}\right)\left(z, y-y^{g}\right), \\
& h_{2}(x, y, u ; g):=\left(u-u^{g}\right)\left(x, y-y^{g}\right)-\left(y-y^{g}\right)\left(x, u-u^{g}\right),
\end{aligned}
$$

where $x, z \in \mathfrak{h}^{*}, y, u \in \mathfrak{h}$. It is clear that $h(x, y, z ; g)$ is antisymmetric in $x, z$, while $h_{2}(x, y, u ; g)$ is antisymmetric in $y, u$. Hence, for any $x, y, z, u$ the functions $h_{1}(x, y, z ; g)$ and $h_{2}(x, y, u ; g)$ belong to the ideal of the scheme $\Psi$. This implies that $h_{1} c=0, h_{2} c=0$, as desired.

Note that if $g \in \Psi(\mathbb{C})$ is not unipotent, and $\lambda \neq 1$ is the nontrivial eigenvalue of $g$ in $\mathfrak{h}^{*}$ then $(y,(1-g) x)=(1-\lambda)\left(y^{\prime}, x^{\prime}\right)$, where $x^{\prime}, y^{\prime}$ are the projections of $x, y$ on the image of $1-g$ along the kernel of $1-g$. Hence, if $G$ is a finite group and $\mathfrak{h}$ is a faithful representation then the algebra of Theorem 3.3 is exactly the rational Cherednik algebra of $[\mathrm{EG}]$.

Thus, algebras $\mathrm{H}_{\kappa}$ with $\kappa$ being of the form (3.4) are natural continuous analogs of rational Cherednik algebras. We will therefore call $\mathrm{H}_{\kappa}$ a continuous Cherednik algebra.

Theorem 3.5. Assume that $\mathfrak{h}$ is an irreducible faithful representation of $G$ of real or complex type (i.e., it has no nonzero invariant skew-symmetric form). If $\mathrm{H}_{\kappa}$ is a continuous Hecke algebra, then it is a continuous Cherednik algebra, i.e. $\kappa$ has the form given in Theorem 3.3

Proof. For the proof we will need the following proposition.

Proposition 3.6. If the PBW property holds for $\mathrm{H}_{\kappa}$, then $\kappa(x, y)=0$ whenever $x, y$ are both in $\mathfrak{h}$, or both in $\mathfrak{h}^{*}$.

Proof. Suppose $x, y \in \mathfrak{h}$. It follows from (2.9) that $(z \mid x, y)=0$ for all $z \in \mathfrak{h}^{*}$. Hence, it remains to show that $\kappa(x, y)$ annihilates the constant functions on $G$. This follows from the identity $\left(\bigwedge^{2} \mathfrak{h}^{*}\right)^{G}=0$. Similarly for $x, y \in \mathfrak{h}^{*}$.

Now let us prove Theorem 3.5. Let $z, x \in \mathfrak{h}^{*}, y \in \mathfrak{h}$. By the Jacobi identity (2.9) and Proposition [3.6. we have

$$
(z \mid y, x)=(x \mid y, z) .
$$

Taking the inner product of both sides with $u \in \mathfrak{h}$, we get

$$
(u,(1-g) z) \kappa(y, x)=(u,(1-g) x) \kappa(y, z) .
$$

On the other hand, we have

$$
\left(u-u^{g^{-1}}\right) \kappa(y, z)=\left(y-y^{g^{-1}}\right) \kappa(u, z) ;
$$


this follows from the Jacobi identity by applying $g^{-1}$. Let us now move $1-g$ to the left in the right hand side of (3.7), and apply (3.8). This gives

$$
(u,(1-g) z) \kappa(y, x)=(y,(1-g) x) \kappa(u, z) .
$$

Let $e_{i}$ be a basis of $\mathfrak{h}, e_{i}^{*}$ the dual basis of $\mathfrak{h}^{*}$. Let $t_{i j}=\left(e_{i},(1-g) e_{j}^{*}\right), c_{i j}=\kappa\left(e_{i}, e_{j}^{*}\right)$. Then (3.9) implies

$$
t_{i j} c_{p q}-t_{p q} c_{i j}=0
$$

Let us regard all our distributions as distributions on $G L(\mathfrak{h})$. We claim that $c_{i j}=t_{i j} b$ for some distribution $b$ on $G L(\mathfrak{h})$. Indeed, by (3.10), $\left\{c_{i j}\right\}$ is a 1-cocycle in the Koszul complex of the $\mathcal{O}(G L(\mathfrak{h}))$-module $\mathcal{O}(G L(\mathfrak{h}))^{*}$ for the sequence of elements $\left\{t_{i j}\right\}$. But this complex is exact, since $\mathcal{O}(G L(\mathfrak{h}))^{*}$ is dual to a free module, and the sequence $\left\{t_{i j}\right\}$ is regular. Thus, $\left\{c_{i j}\right\}$ is a coboundary of some element $b$, as desired.

Thus we have

$$
\kappa(x, y)=(y,(1-g) x) b
$$

The distribution $b$ is defined up to adding a multiple of $\delta_{1}$, so it is invariant under $G$.

Since $\kappa$ is supported on $G$, we find that $(y,(1-g) x) b$ is supported on $G$ for all $x, y$. Thus, for any function $\phi$ vanishing on $G, b \phi=\gamma(\phi) \delta_{1}$, where $\gamma$ is a scalar-valued $G$ invariant linear functional such that $\gamma(\phi f)=\gamma(\phi) f(1), f \in \mathcal{O}(G L(\mathfrak{h}))$. This means that $\gamma$ has the form $\gamma(\phi)=<(d \phi)(1), Y>$, where $Y \in \mathfrak{g l}(\mathfrak{h})$ is an invariant element (i.e., $Y=t \cdot \operatorname{Id}$ is a scalar operator, since $\mathfrak{h}$ is irreducible). Let $c=b-Y$. Then we get

$$
\kappa(x, y)=t(y, x) \delta_{1}+(y,(1-g) x) c,
$$

and $c$ is supported on $G$.

It remains to show that $c$ is (scheme-theoretically) supported on $\Psi$. But this is clear from the Jacobi identity:

$$
c(y,(1-g) x)(1-g) z=c(y,(1-g) z)(1-g) x .
$$

The theorem is proved.

Corollary 3.11. If $\mathfrak{h}$ is a faithful irreducible representation of real or complex type, then any continuous symplectic reflection algebra is a continuous Cherednik algebra, and vice versa.

Proof. It follows from Theorems 3.1] 3.5 that any continuous symplectic reflection algebra is a continuous Cherednik algebra. To prove the converse, let $e_{i}$ be a basis of $\mathfrak{h}$ and $e_{i}^{*}$ the dual basis of $\mathfrak{h}^{*}$. Then on $\Psi$ we have

$\omega((1-g) x,(1-g) y)=\sum_{i}\left((1-g) x, e_{i}\right)\left(e_{i}^{*},(1-g) y\right)=-\sum_{i}\left(g x,(1-g) e_{i}\right)\left(e_{i}^{*},(1-g) y\right)=$ 


$$
-\sum_{i}(g x,(1-g) y)\left(e_{i}^{*},(1-g) e_{i}\right)=\sum_{i}((1-g) x, y)\left(e_{i}^{*},(1-g) e_{i}\right)=\phi(g)(y,(1-g) x),
$$

where $\phi(g)=\left.\operatorname{Tr}(1-g)\right|_{\mathfrak{h}}$. Now let $c$ be a $G$-invariant distribution on $\Psi$, and $\widetilde{c}$ be a $G$ invariant distribution on $G$ such that $\phi \widetilde{c}=c$ (it exists since $\phi$ is not a zero divisor in $\mathcal{O}(G)^{G}$, as for any element $g \neq 1$ of a maximal compact subgroup of $G$, one has $\phi(g)>0)$, so the multiplication operator by $\phi$ is injective on $\mathcal{O}(G)^{G}$ and hence surjective on $\left.\left(\mathcal{O}(G)^{G}\right)^{*}\right)$. Then we have

$$
\omega((1-g) x,(1-g) y) \widetilde{c}=(y,(1-g) x) c .
$$

From the Jacobi identity it is clear that $\widetilde{c}$ is supported on $\Sigma$, which implies the required statement.

Corollary 3.12. Let $(\mathbf{G}, \mathbf{K})$ be a Hermitian symmetric pair (with semisimple $\mathbf{G})$. Then $A(\mathbf{G}, \mathbf{K})$ is a continuous Cherednik algebra.

Proof. Let $\mathfrak{g}, \mathfrak{k}$ be the Lie algebras of $\mathbf{G}, \mathbf{K}$, Then $\mathfrak{g}=\mathfrak{k} \oplus \mathfrak{p}_{+} \oplus \mathfrak{p}_{-}$, and we have a linear representation $\mathbf{K} \rightarrow G L\left(\mathfrak{p}_{+}\right)$Also, we have $\left[\mathfrak{p}_{+}, \mathfrak{p}_{+}\right]=\left[\mathfrak{p}_{-}, \mathfrak{p}_{-}\right]=0$, while $\left[\mathfrak{p}_{+}, \mathfrak{p}_{-}\right] \subset \mathfrak{k}$. It follows (after a simple computation) that $A(\mathbf{G}, \mathbf{K})=\mathbf{H}_{\kappa}(\mathbf{K})$, where $\kappa$ is such that $t=0$ and $c=\Delta \delta_{1}, \Delta$ being the Laplacian on $\mathbf{K}$ attached to the Killing form of $\mathbf{G}$ restricted to K.

Remark. We see that it is possible that the scheme $\Psi / G$ is not reduced (i.e., $\Psi / G \neq$ $S / G)$, and hence $\mathrm{C}(S) \neq \mathrm{C}(\Psi)$. Indeed, for Hermitian symmetric pairs one often has $S=\{1\}$, and yet one has a distribution $\kappa(x, y)$ which satisfies the Jacobi identity and is not a multiple of $\delta_{1}$.

\subsection{Examples.}

\subsubsection{The case $G=G L_{n}$.}

Let $\mathfrak{h}$ be an $n$ dimensional vector space, and let $G=G L_{n}$ be the general linear group of $\mathfrak{h}$. In this case, we have an isomorphism $S / G \rightarrow \mathbb{C}^{*}: s \mapsto \lambda=\left.\operatorname{Tr}\right|_{\mathfrak{h}}(s)-n+1$. Thus, $\mathrm{C}(S)$ is the space of Fourier series $\sum_{m \in \mathbb{Z}} c_{m} \lambda^{m}$. Also, the scheme $\Psi$ is well known to be reduced, so we have $\Psi=S$. Thus in this case $\mathrm{C}(\Psi)=\mathrm{C}(S)$.

\subsubsection{The case $G=O_{n}$.}

Let $\mathfrak{h}$ be an $n$ dimensional vector space with a nondegenerate symmetric bilinear form, and let $G=O_{n}$ be the orthogonal group of $\mathfrak{h}$. Note that for $n=2$ this example can be regarded as a continuous limit of symplectic reflection algebras of finite dihedral groups. 
In this case $S$ consists of 1 and the conjugacy class of orthogonal reflections, so $S / G$ consists of two points and hence the space $\mathrm{C}(S)$ is two dimensional. However, it turns out that $\Psi$ is not reduced in this case. Luckily, we have the following proposition.

Proposition 3.13. For $G=O_{n}, \mathrm{C}(\Psi)=\mathrm{C}(S)$.

Proof. First we claim that $\Psi$ is reduced near every orthogonal reflection. Indeed, let $s=$ $\operatorname{diag}(-1,1, \ldots, 1) \in S$. Then the tangent space $T_{s} S$ is the space $[s, \mathfrak{g}]$ of symmetric matrices $A=\left(a_{i j}\right)$ such that $a_{i j}=0$ unless $i \neq j$ and $i=1$ or $j=1$. Now assume that $s+\hbar A$ is a point of $\Psi$ over the ring of dual numbers $\mathbb{C}[\hbar] / \hbar^{2}$. The nontrivial minors of $1-s-\hbar A$ are $\Delta_{1 i, 1 j}=-2 \hbar a_{i j}$, so we get $a_{i j}=0$ if $i, j>1$. We also have $\operatorname{det}(s+\hbar A)=-1+\hbar a_{11}$, so $a_{11}=0$. Hence, $A \in T_{s} S$, and thus $T_{s} \Psi=T_{s} S$.

It remains to consider the component $\Psi_{1}$ of the identity in $\Psi$. Writing $g=e^{A}$, where $A$ is skew-symmetric, we may ( $G$-equivariantly) identify $\Psi_{1}$ with the subscheme of $\mathfrak{s o}_{n}$ defined by the equation $\wedge^{2} A=0$.

We claim that the ring $\mathcal{O}\left(\Psi_{1}\right)$ is spanned by functions 1 and $a_{i j}, i<j$, with trivial multiplication. Indeed, it is easy to see that $1, a_{i j}$ are linearly independent, and $a_{i j} a_{k i}=a_{i j} a_{k i}-a_{i i} a_{k j}=0$. Also, $a_{i j} a_{k l}$ is symmetric under the transposition of $j, l$ but antisymmetric under the transposition of $i, j$ and $k, l$, so it is zero.

Thus, $\mathcal{O}\left(\Psi_{1}\right)=\mathbb{C} \oplus \wedge^{2} \mathfrak{h}$ as a $G$-module, and hence $\mathcal{O}\left(\Psi_{1} / G\right)=\mathbb{C}$, as desired.

We conclude that for $G=O_{n}$ the continuous Cherednik algebras form a 2-parameter family (out of which only one is essential, because of scaling).

\subsubsection{The case $G=S p_{2 n}$.}

Let $V$ be a $2 n$ dimensional vector space with a nondegenerate skew-symmetric bilinear form, and let $G=S p_{2 n}$ be the symplectic group of $V$. We have an isomorphism $S / G \rightarrow$ $\mathbb{C}: s \mapsto w=\lambda+\lambda^{-1}=\left.\operatorname{Tr}\right|_{V}(s)-2 n+2$. Thus, $\mathrm{C}(S)$ is the space of Fourier series $\sum_{m \in \mathbb{Z}} c_{m} \lambda^{m}$ with $c_{m}=c_{-m}$.

Theorem 3.14. We have: (i) $\Phi=S$, and $S$ is an irreducible affine algebraic variety;

(ii) if the $P B W$ property holds for $\mathrm{H}_{\kappa}$, then $\kappa(x, y)$ is of the form (3.2), with $c \in$ $\mathrm{C}(\Phi)=\mathrm{C}(S)$.

Proof. (i) We first show that $\Phi$ is reduced (i.e., coincides with $S$ ). Let $\bar{S} \subset S$ be the subset of elements which are not unipotent. Let $\operatorname{Gr}(2 n-2,2 n)$ be the Grassmannian variety of $2 n-2$ dimensional subspaces in $V$. The map $\bar{S} \rightarrow G r(2 n-2,2 n): g \mapsto \operatorname{Ker}(1-g)$ is a smooth submersion, with open image (the set of nondegenerate codimension 2 subspaces of the symplectic space $V$ ). Hence $\bar{S}$ is smooth. 
Any conjugacy class in $\bar{S}$ contains a representative of the form

$$
g=\left(\begin{array}{ccccc}
a & b & & & \\
c & d & & & \\
& & 1 & & \\
& & & \ddots & \\
& & & & 1
\end{array}\right)
$$

We claim that the tangent spaces $T_{g} \Phi$ and $T_{g} S$ are equal. Indeed, let $g+\hbar Q\left(Q=\left(q_{i j}\right)\right)$ be a point of $\Phi$ over the ring $\mathbb{C}[\hbar] / \hbar^{2}$. Then we have $\operatorname{Tr}\left(g^{-1} Q\right)=0$, and the equations $\wedge^{3}(g+\hbar Q-1)=0$ imply that $q_{i j}=0$ for all $i, j \geq 3$. On the other hand, $T_{g} S$ is spanned by matrices of the form $[g, M]$ where $M \in \mathfrak{s p}(2 n)$, and matrices $Q=g P$, where $P$ has the form

$$
\left(\begin{array}{ccccc}
\alpha & \beta & & & \\
\gamma & \delta & & & \\
& & 0 & & \\
& & & \ddots & \\
& & & & 0
\end{array}\right) \text { where } \alpha+\delta=0
$$

Therefore, $T_{g} \Phi=T_{g} S$, and so $\Phi$ is reduced at the formal neighborhood of each $g \in \bar{S}$.

The set of points of $S$ where $\Phi$ is not reduced is $G$-invariant. Therefore, to prove that this set is empty, it remains to show that $\Phi$ is reduced at the formal neighborhood of 1 . Let $A$ be the algebra of functions on the formal neighborhood of 1 in the scheme $\wedge^{3}(1-g)=0$, where $g \in G$. Let $A_{0}$ be the algebra of functions on the formal neighborhood of 0 in the scheme $\wedge^{3} M=0$, where $M \in \mathfrak{s p}(2 n)$. Explicitly, $A=\mathbb{C}[[1-g]] /\left(\wedge^{3}(1-g)\right)$ and $A_{0}=\mathbb{C}[[M]] /\left(\wedge^{3} M\right)$, where $g=\left(g_{i j}\right)$ and $M=\left(m_{i j}\right)$ are matrices of formal variables. The pullback of the exponential map gives an isomorphism exp* $: \mathbb{C}[[1-g]] \rightarrow \mathbb{C}[[M]]$ : $g \mapsto 1+M+M^{2} / 2 !+\cdots$. Now $\exp ^{*}: \wedge^{3}(g-1) \mapsto \wedge^{3}\left(M\left(1+M / 2 !+M^{2} / 3 !+\cdots\right)\right)$. The element $1+M / 2 !+M^{2} / 3 !+\cdots$ is invertible, and hence exp* gives an isomorphism from $A$ to $A_{0}$. By [Ku, Theorem 1], $A_{0}$ is reduced, so $A$ is also reduced.

The irreducibility of $\Phi=S$ follows from the easy fact from linear algebra that $\bar{S}$ (which is irreducible) is a dense subset of $S$.

(ii) Let $S^{\prime}$ be the subset of elements of $S$ which are not unipotent and do not have eigenvalue -1 . By part (i), $S$ is irreducible, and $S^{\prime}$ is an affine open set in $S$, defined by the condition $f^{2}-4 \neq 0$, where $f(s)=\operatorname{Tr}(s)-2 n+2$. Hence $\mathcal{O}(S) \subset \mathcal{O}\left(S^{\prime}\right)$. Thus, we have a surjective $G$-invariant $\operatorname{map} \xi: \mathcal{O}\left(S^{\prime}\right)^{*} \rightarrow \mathcal{O}(S)^{*}$.

The group $G$ acts on $S^{\prime}$, with $S^{\prime} / G=\mathbb{C} \backslash\{2,-2\}$, and the stabilizer of each point is isomorphic to $K=S p(2 n-2) \times \mathbb{C}^{*}$. Thus, $S^{\prime}$ is a fibration over $\mathbb{C} \backslash\{2,-2\}$ whose fiber is $G / K$, and the action of $G$ is fiberwise.

Let $\kappa$ be a distribution satisfying the PBW property. Then, as follows from Proposition 2.8 and part (i), $\kappa \in\left(\mathcal{O}(S)^{*} \otimes \wedge^{2} V^{*}\right)^{G}$. Let $\bar{\kappa}$ be a lifting of $\kappa$ in $\left(\mathcal{O}\left(S^{\prime}\right)^{*} \otimes \wedge^{2} V^{*}\right)^{G}$. Since 
$S^{\prime}$ is fibered over $\mathbb{C} \backslash\{2,-2\}$ with fiber $G / K$, we may view $\bar{\kappa}$ as a distribution on $\mathbb{C} \backslash\{2,-2\}$ with values in the space $\left(\wedge^{2} V^{*}\right)^{K}$, which for $n>1$ is a two dimensional space spanned by $\omega(x, y)$ and $\omega((1-g) x,(1-g) y)$ (where $g$ is a semisimple element in $S$ whose centralizer in $G$ is $K)$. In other words, we have

$$
\bar{\kappa}(g)(x, y)=\alpha(g) \omega(x, y)+\beta(g) \omega((1-g) x,(1-g) y),
$$

where $\alpha, \beta$ are $G$-invariant distributions on $S^{\prime}$. The Jacobi identity for $\kappa$ implies that

$$
\left(z-z^{g}\right) \bar{\kappa}(x, y)+\left(x-x^{g}\right) \bar{\kappa}(y, z)+\left(y-y^{g}\right) \bar{\kappa}(z, x)=0
$$

modulo distributions that vanish on $\mathcal{O}(S)$. This imposes no condition on $\beta$, and the condition on $\alpha$ is that the distribution

$$
\alpha(g)\left(\left(z-z^{g}\right) \omega(x, y)+\left(x-x^{g}\right) \omega(y, z)+\left(y-y^{g}\right) \omega(z, x)\right)
$$

vanishes on $\mathcal{O}(S)$. This implies that $\alpha$ is scheme-theoretically supported at 1 , so $\alpha=t \delta_{1}$. The result follows.

If $n=1$, then the proof is even simpler, since in this case $\left(\wedge^{2} V^{*}\right)^{K}$ is a 1-dimensional space spanned by $\omega$.

\section{Infinitesimal Hecke algebras}

4.1 The main theorem. Let $\mathfrak{g}$ be the Lie algebra of $G$. The universal enveloping algebra $\mathcal{U} \mathfrak{g}$ is naturally isomorphic to the subalgebra of $\mathcal{O}(G)^{*}$ consisting of all algebraic distributions set-theoretically supported at the identity element 1 of $G$, cf. [DG, II, §6]. When the image of $\kappa: V \times V \rightarrow \mathcal{O}(G)^{*}$ lies in $\mathcal{U} \mathfrak{g}$, let $\mathscr{H}_{\kappa}=\mathscr{H}_{\kappa}(\mathfrak{g})$ be the quotient of $T V \rtimes \mathcal{U} \mathfrak{g}$ by the relations (3.2).

We define a filtration on $\mathscr{H}_{\kappa}$ by setting $\operatorname{deg}(x)=1, \operatorname{deg}(u)=0$ for $x \in V, u \in$ $\mathcal{U} \mathfrak{g}$. There is a natural map $\mathscr{H}_{0}=S V \rtimes \mathcal{U} \mathfrak{g} \rightarrow \operatorname{gr}\left(\mathscr{H}_{\kappa}\right)$ which is a surjective graded algebra homomorphism, and we say that the PBW property holds for $\mathscr{H}_{\kappa}$ if this map is an isomorphism. Since $\mathrm{H}_{\kappa}(G)=\mathcal{O}(G)^{*} \otimes \mathcal{U}_{\mathfrak{g}} \mathscr{H}_{\kappa}(\mathfrak{g})$, the PBW property holds for $\mathscr{H}_{\kappa}(\mathfrak{g})$ if and only if it holds for $\mathrm{H}_{\kappa}(G)$, i.e. when $\kappa$ satisfies the Jacobi identity. Whenever this is the case, we call $\mathscr{H}_{\kappa}$ an infinitesimal Hecke algebra.

Example 4.1. For instance, we can define infiniteimal Hecke algebras $\mathscr{H}_{\kappa}$ in the examples attached to symmetric pairs, see Section 2.4 in this case it is easy to see that $\mathscr{H}_{\kappa}$ is the universal enveloping algebra of the Lie algebra of the group $\mathbf{G}$.

The aim of this section is to prove the following result when $\mathfrak{g}$ is $\mathfrak{g l}_{n}$ or $\mathfrak{s p}_{2 n}$.

Theorem 4.2. The PBW property holds for $\mathscr{H}_{\kappa}(\mathfrak{g})$ (i.e., it is an infinitesimal Hecke algebra) if and only if the relations (2.3) are of the form (4.4) when $\mathfrak{g}=\mathfrak{g l}_{n}$, and (4.9) when $\mathfrak{g}=\mathfrak{s p}_{2 n}$.

The relations (4.4) and (4.9) will be given below. 


\subsubsection{The $\mathfrak{g}=\mathfrak{g l}_{n}$ case.}

Let $\mathfrak{h}:=\mathbb{C}^{n}, V:=\mathfrak{h} \oplus \mathfrak{h}^{*}$ and $\mathfrak{g}:=\mathfrak{g l}_{n}=\mathfrak{g l}(\mathfrak{h})$. We shall identify $\mathfrak{g}^{*}$ with $\mathfrak{g}$ via the pairing $\mathfrak{g} \times \mathfrak{g} \rightarrow \mathbb{C}:(A, B) \mapsto \operatorname{Tr}(A B)$. We shall also identify $S \mathfrak{g}$ with $\mathcal{U} \mathfrak{g}$ via the symmetrization map. For any $x \in \mathfrak{h}^{*}, y \in \mathfrak{h}, A \in \mathfrak{g}$, we may write

$$
\left(x,(1-\tau A)^{-1} y\right) \operatorname{det}(1-\tau A)^{-1}=r_{0}(x, y)(A)+r_{1}(x, y)(A) \tau+r_{2}(x, y)(A) \tau^{2}+\cdots
$$

where $r_{i}(x, y) \in S \mathfrak{g}$ is a polynomial function on $\mathfrak{g}$.

For each polynomial $\beta=\beta_{0}+\beta_{1} \tau+\beta_{2} \tau^{2}+\cdots \in \mathbb{C}[\tau]$, define the algebra $\mathscr{H}_{\beta}=$ $\mathscr{H}_{\beta}\left(\mathfrak{g l}_{n}(\mathbb{C})\right)$ to be the quotient of $T\left(\mathfrak{h} \oplus \mathfrak{h}^{*}\right) \rtimes \mathcal{U} \mathfrak{g}$ by the relations

$$
\left[x, x^{\prime}\right]=0, \quad\left[y, y^{\prime}\right]=0, \quad[y, x]=\beta_{0} r_{0}(x, y)+\beta_{1} r_{1}(x, y)+\beta_{2} r_{2}(x, y)+\cdots,
$$

for all $x, x^{\prime} \in \mathfrak{h}^{*}$ and $y, y^{\prime} \in \mathfrak{h}$.

Remark 4.5. To expand the left hand side of (4.3) as a power series in $\tau$, we write

$$
\begin{gathered}
(1-\tau A)^{-1}=1+\tau A+\tau^{2} A^{2}+\cdots, \\
\operatorname{det}(1-\tau A)^{-1}=e^{-\log \operatorname{det}(1-\tau A)}=e^{-\operatorname{Tr} \log (1-\tau A)}=e^{\sum_{k>0} \tau^{k} \operatorname{Tr}\left(A^{k} / k\right)} \\
=1+\tau \operatorname{Tr}(A)+\tau^{2}\left(\operatorname{Tr}\left(\frac{A^{2}}{2}\right)+\frac{(\operatorname{Tr} A)^{2}}{2}\right)+\cdots .
\end{gathered}
$$

Example 4.6. We have $r_{0}(x, y)=(x, y)$. Thus, $\mathscr{H}_{1}\left(\mathfrak{g l}_{n}(\mathbb{C})\right)=D\left(\mathbb{C}^{n}\right) \rtimes \mathcal{U g l}_{n}(\mathbb{C})$ (where $D\left(\mathbb{C}^{n}\right)$ is the algebra of polynomial differential operators on $\left.\mathbb{C}^{n}\right)$.

Example 4.7. We have $r_{1}(x, y)=y \otimes x+(x, y) \operatorname{Id} \in \mathfrak{g l}_{n}(\mathbb{C})$. Thus, $\mathscr{H}_{\tau}\left(\mathfrak{g l}_{n}(\mathbb{C})\right)=$ $\mathcal{U}\left(\mathfrak{h} \oplus \mathfrak{h}^{*} \oplus \mathfrak{g l}_{n}(\mathbb{C})\right)$, where the Lie bracket on $\mathfrak{h} \oplus \mathfrak{h}^{*} \oplus \mathfrak{g l}_{n}(\mathbb{C})$ is

$$
\left[(y, x, A),\left(y^{\prime}, x^{\prime}, A^{\prime}\right)\right]=\left(A y^{\prime}-A^{\prime} y, x A^{\prime}-x^{\prime} A,\left[A, A^{\prime}\right]+r_{1}\left(x^{\prime}, y\right)-r_{1}\left(x, y^{\prime}\right)\right) .
$$


There is a Lie algebra isomorphism $\varphi: \mathfrak{h} \oplus \mathfrak{h}^{*} \oplus \mathfrak{g l}_{n}(\mathbb{C}) \rightarrow \mathfrak{s l}_{n+1}(\mathbb{C})$ defined by

$$
\begin{aligned}
& y \mapsto\left(\begin{array}{c|c}
0 & y \\
\hline 0 & 0
\end{array}\right) \quad \text { if } y \in \mathfrak{h}, \\
& x \mapsto\left(\begin{array}{c|c}
0 & 0 \\
& \\
\hline-x & 0
\end{array}\right) \quad \text { if } x \in \mathfrak{h}^{*}, \\
& A \mapsto\left(\begin{array}{c|c}
A & 0 \\
& \\
\hline 0 & 0
\end{array}\right) \quad \text { if } A \in \mathfrak{s l}_{n}(\mathbb{C}) \subset \mathfrak{g l}_{n}(\mathbb{C}) \text {, } \\
& \lambda \operatorname{Id} \mapsto\left(\begin{array}{ccc|c}
\frac{\lambda}{n+1} & & & \\
& \ddots & & 0 \\
& & \frac{\lambda}{n+1} & \\
\hline 0 & & \frac{-n \lambda}{n+1}
\end{array}\right) \quad \text { if } \lambda \in \mathbb{C} .
\end{aligned}
$$

Obviously, this example comes from the hermitian symmetric pair $\left(S L_{n+1}, G L_{n}\right)$, as explained in Section 2.4

\subsubsection{The $\mathfrak{g}=\mathfrak{s p}_{2 n}$ case.}

Let $V=\mathbb{C}^{2 n}$ be a symplectic vector space with symplectic form $\omega$, and $\mathfrak{g}:=\mathfrak{s p}(V)$. Again, we identify $\mathfrak{g}^{*}$ with $\mathfrak{g}$ via the pairing $\mathfrak{g} \times \mathfrak{g} \rightarrow \mathbb{C}:(A, B) \mapsto \operatorname{Tr}(A B)$, and $S \mathfrak{g}$ with $\mathcal{U} \mathfrak{g}$ via the symmetrization map. For any $x, y \in V, A \in \mathfrak{g}$, we may write

$$
\omega\left(x,\left(1-\tau^{2} A^{2}\right)^{-1} y\right) \operatorname{det}(1-\tau A)^{-1}=\ell_{0}(x, y)(A)+\ell_{2}(x, y)(A) \tau^{2}+\ell_{4}(x, y)(A) \tau^{4}+\cdots
$$

where $\ell_{i}(x, y) \in S \mathfrak{g}$ is a polynomial function on $\mathfrak{g}$.

For each polynomial $\beta=\beta_{0}+\beta_{2} \tau^{2}+\beta_{4} t^{4}+\cdots \in \mathbb{C}[\tau]$, define the algebra $\mathscr{H}_{\beta}=$ $\mathscr{H}_{\beta}(\mathfrak{s p}(V))$ to be the quotient of $T V \rtimes \mathcal{U} \mathfrak{g}$ by the relations

$$
[x, y]=\beta_{0} \ell_{0}(x, y)+\beta_{2} \ell_{2}(x, y)+\beta_{4} \ell_{4}(x, y)+\cdots,
$$

for all $x, y \in V$.

Remark 4.10. Note that $A \in \mathfrak{s p}(V)$ implies $A^{k}=(-1)^{k} J^{T}\left(A^{T}\right)^{k} J$, where $J$ is the matrix of the form $\omega$ in a symplectic basis. Thus, in (4.8), $\omega\left(x,\left(1-\tau^{2} A^{2}\right)^{-1} y\right)$ is skew-symmetric in $x, y$. We also have $\operatorname{Tr}\left(A^{k}\right)=0$ if $k$ is odd, so the expansion of $\operatorname{det}(1-\tau A)^{-1}$ contains only even powers of $\tau$. 
Example 4.11. We have $\ell_{0}(x, y)=\omega(x, y)$. Thus, $\mathscr{H}_{1}(\mathfrak{s p}(V))=A_{n} \rtimes \mathcal{U} \mathfrak{s p}(V)$, where $A_{n}$ is the $n$-th Weyl algebra.

Example 4.12. When $n=1$, we have $\mathfrak{g}=\mathfrak{s l}_{2}$. Let

$$
A=\left(\begin{array}{rr}
a & b \\
c & -a
\end{array}\right), e=\left(\begin{array}{ll}
0 & 1 \\
0 & 0
\end{array}\right), h=\left(\begin{array}{rr}
1 & 0 \\
0 & -1
\end{array}\right), f=\left(\begin{array}{ll}
0 & 0 \\
1 & 0
\end{array}\right) .
$$

Note that $A^{2}=\left(a^{2}+b c\right) I d$, so $\ell_{2 k}(x, y)$ is equal to a constant times $\omega(x, y) \Delta^{k}$, where $\Delta=\frac{h^{2}}{2}+e f+f e$ is the Casimir element of $\mathcal{U}_{\mathfrak{s l}}$. The algebra $\mathscr{H}_{\beta}\left(\mathfrak{s l}_{2}\right)$ was studied by Khare in $\mathrm{Kh}$.

4.2 Proof of Theorem 4.2. Let $G=G L_{n}$ when $\mathfrak{g}=\mathfrak{g l}_{n}$, and let $G=S p_{2 n}$ when $\mathfrak{g}=\mathfrak{s p}_{2 n}$. By Theorems 3.1, 3.14, 3.5, and Corollary 3.11, the PBW property holds if and only if $\kappa$ is of the form (3.2). Thus we have to find all $c \in \mathrm{C}(S)$ such that $\kappa(x, y)$ is set-theoretically supported at 1 for all $x, y \in V$, and then compute $\kappa(x, y)$ as an element in $\mathcal{U} \mathfrak{g}$.

The $\mathfrak{g}=\mathfrak{g l}_{n}$ case: By Proposition 3.6. we may assume that $\kappa(x, y)=0$ if $x, y$ are both in $\mathfrak{h}$ or both in $\mathfrak{h}^{*}$. Following Subsection 3.3.1 we identify $\mathrm{C}(S)$ with the space of algebraic distributions on $\mathbb{C}^{*}$, so that $c \in \mathbb{C}\left[\lambda, \frac{1}{\lambda}\right]^{*}$. Then $\kappa(x, y)$ is set-theoretically supported at 1 for all $x, y \in V$ if and only if $c=c(\lambda)$ is a finite linear combination of $\delta_{1}$ and its derivatives.

Now let $\mathfrak{h}=\mathbb{C}^{n}$ be equipped with the Hermitian inner product $\langle x, y\rangle:=x^{T} \bar{y}$. We may identify $\mathfrak{h}^{*}$ with $\overline{\mathfrak{h}}$ using this inner product on $\mathfrak{h}$. Let

$$
\begin{gathered}
K:=U(n, \mathbb{C})=\left\{g \in G, \mid g^{-1}=\bar{g}^{T}\right\}, \\
s_{\theta}:=\operatorname{diag}\left(e^{i \theta}, 1, \ldots, 1\right) \in K, \quad \theta \in[0,2 \pi], \\
S_{\theta}:=\left\{g s_{\theta} g^{-1} \mid g \in K\right\} \subset K .
\end{gathered}
$$

For any $s \in S_{\theta}$, denote by $p_{s} \in \mathfrak{g}$ the orthogonal projection of $\mathfrak{h}$ to the eigenspace of $s$ with eigenvalue $e^{i \theta}$. We have $p_{s}=v \otimes \bar{v}$ where $v \in \mathfrak{h}$ is an eigenvector of $s$ with eigenvalue $e^{i \theta}$ and $|v|=1$. We have to compute

$$
\begin{aligned}
\kappa(x, y) & =t(x, y)+\int_{0}^{2 \pi} c(\theta)\left(\int_{S_{\theta}}\left(1-e^{-i \theta}\right)\left(x, p_{s} y\right) s d s\right) d \theta \\
& =t(x, y)+\int_{|v|=1}(x,(v \otimes \bar{v}) y)\left(\int_{0}^{2 \pi}\left(1-e^{-i \theta}\right) c(\theta) e^{i \theta(v \otimes \bar{v})} d \theta\right) d v \\
& =t(x, y)+\int_{|v|=1}(x,(v \otimes \bar{v}) y) f(v \otimes \bar{v}) d v \in \mathcal{U} \mathfrak{g},
\end{aligned}
$$

where $x \in \mathfrak{h}^{*}, y \in \mathfrak{h}, c(\theta)$ is a finite linear combination of $\delta_{0}$ and its derivatives, and $f$ is some polynomial depending on $c$. 
Recall that we identify $\mathcal{U} \mathfrak{g}$ with $S \mathfrak{g}$ via the symmetrization map. Let $F_{m} \in S \mathfrak{g}$ be the function on $\mathfrak{g}$ defined by

$$
F_{m}(A):=\int_{|v|=1}\langle A v, v\rangle^{m+1} d v, \quad A \in \mathfrak{g} .
$$

Note that

$$
\left.d F_{m}\right|_{A}(y \otimes x)=(m+1) \int_{|v|=1}(x,(v \otimes \bar{v}) y)\langle A v, v\rangle^{m} d v .
$$

Thus $\kappa(x, y)$ corresponds, under symmetrization map, to a finite linear combination of functions $\left.d F_{m}\right|_{A}(y \otimes x)$.

We now compute $F_{m}(A)$. First, note that

$$
\begin{aligned}
\int_{\mathfrak{h}}\langle A v, v\rangle^{m+1} e^{-\zeta\langle v, v\rangle} d v & =\int_{0}^{\infty} e^{-\zeta r^{2}} \int_{|v|=r}\langle A v, v\rangle^{m+1} d v d r \\
& =\int_{0}^{\infty} r^{2 m+2 n+1} e^{-\zeta r^{2}} d r \cdot F_{m}(A) \\
& =\frac{(m+n) !}{2} \zeta^{-m-n-1} F_{m}(A) .
\end{aligned}
$$

On the other hand, we have

$$
\begin{aligned}
\sum_{m=-1}^{\infty} \frac{1}{(m+1) !} \int_{\mathfrak{h}}\langle A v, v\rangle^{m+1} e^{-\zeta\langle v, v\rangle} d v & =\int_{\mathfrak{h}} e^{\langle A v, v\rangle-\zeta\langle v, v\rangle} d v \\
& =\int_{\mathfrak{h}} e^{-\langle(\zeta-A) v, v\rangle} d v \\
& =\pi^{n} \operatorname{det}(\zeta-A)^{-1} .
\end{aligned}
$$

Hence, $F_{m}(A)$ is equal to a constant times the coefficient of $\tau^{m+1}$ in the expansion of $\operatorname{det}(1-\tau A)^{-1}$ as a power series in $\tau=\zeta^{-1}$. Differentiating $\operatorname{det}(1-\tau A)^{-1}$ and evaluating at $B \in \mathfrak{g}$, we obtain

$$
\frac{\partial}{\partial B} \operatorname{det}(1-\tau A)^{-1}=\frac{\operatorname{Tr}\left(\tau B(1-\tau A)^{-1}\right)}{\operatorname{det}(1-\tau A)} .
$$

Setting $B=y \otimes x$ gives $\tau\left(x,(1-\tau A)^{-1} y\right) \operatorname{det}(1-\tau A)^{-1}$, as desired.

The $\mathfrak{g}=\mathfrak{s p}_{2 n}$ case: We let $V=\mathbb{C}^{2 n}$ be equipped with the symplectic form $\omega(x, y)=$ $x^{T} J y$ where

$$
J:=\left(\begin{array}{ccccc}
0 & 1 & & & \\
-1 & 0 & & & \\
& & 0 & 1 & \\
& & -1 & 0 & \\
& & & & \ddots
\end{array}\right)
$$


Let

$$
\begin{gathered}
K:=U(n, \mathbb{H})=\left\{g \in G L_{2 n}(\mathbb{C}) \mid g^{T} J g=J, g^{-1}=\bar{g}^{T}\right\}, \\
s_{\theta}:=\operatorname{diag}\left(e^{i \theta}, e^{-i \theta}, 1, \ldots, 1\right) \in K, \quad \theta \in[0,2 \pi], \\
S_{\theta}:=\left\{g s_{\theta} g^{-1} \mid g \in K\right\} \subset K .
\end{gathered}
$$

(here $\mathbb{H}$ is the division algebra of quaternions ${ }^{5}$. For any $s \in S_{\theta}$, we denote by $p_{s}$ the $\omega$-orthogonal projection of $V$ to $\operatorname{Im}(1-s)$.

Notice that if $s \in S_{\theta}, q \in V$, and $s q=e^{i \theta} q$, then $s J \bar{q}=J \overline{s q}=e^{-i \theta} J \bar{q}$. Take any $q \in V$ with $q^{T} \bar{q}=1$. For any $y \in V$, we have $y=\omega(J \bar{q}, y) q+\omega(y, q) J \bar{q}+y^{\prime}$, where $y^{\prime}$ is $\omega$-orthogonal to $q$ and $J \bar{q}$. Let

$$
s_{q}(\theta):=e^{i \theta p_{q}}, \text { where } p_{q}:=q \bar{q}^{T}+J \bar{q} q^{T} J \in i \operatorname{Lie} K .
$$

Note that if $q=(1,0, \ldots, 0)^{T}$, then $p_{q}=\operatorname{diag}(1,-1,0, \ldots, 0)$ and $s_{q}(\theta)=s_{\theta}$.

Let $x, y \in V$. Now

$$
\begin{aligned}
\omega\left(x, p_{s_{q}(\theta)} y\right) & =\omega(x, \omega(J \bar{q}, y) q+\omega(y, q) J \bar{q}) \\
& =\omega(x, q) \omega(J \bar{q}, y)-\omega(y, q) \omega(J \bar{q}, x) \\
& =\bar{q}^{T}\left(y x^{T}-x y^{T}\right) J q \\
& =\omega\left(\left(x y^{T}-y x^{T}\right) \bar{q}, q\right) .
\end{aligned}
$$

According to Subsection 3.3 .3 we have to compute

$$
\begin{aligned}
\kappa(x, y) & =t \omega(x, y)+\int_{0}^{2 \pi}\left(1-e^{i \theta}\right)\left(1-e^{-i \theta}\right) c(\theta)\left(\int_{S_{\theta}} \omega\left(x, p_{s} y\right) s d s\right) d \theta \\
& =t \omega(x, y)+\int_{|q|=1} \omega\left(\left(x y^{T}-y x^{T}\right) \bar{q}, q\right)\left(\int_{0}^{2 \pi}\left(1-e^{i \theta}\right)\left(1-e^{-i \theta}\right) c(\theta) e^{i \theta p_{q}} d \theta\right) d q \\
& =t \omega(x, y)+\int_{|q|=1} \omega\left(\left(x y^{T}-y x^{T}\right) \bar{q}, q\right) f\left(p_{q}\right) d q \in \mathcal{U} \mathfrak{g},
\end{aligned}
$$

where $c(\theta)$ is a finite linear combination of $\delta_{0}$ and its derivatives (of even order), and $f$ is some polynomial.

Recall that we identify $\mathcal{U} \mathfrak{g}$ with $S \mathfrak{g}$ via the symmetrization map. Let $F_{m}^{\prime} \in S \mathfrak{g}$ be the function on $\mathfrak{g}$ defined by

$$
F_{m}^{\prime}(A):=\int_{|q|=1} \omega\left(\left(x y^{T}-y x^{T}\right) \bar{q}, q\right) \operatorname{Tr}\left(A p_{q}\right)^{m} d q, \quad A \in \mathfrak{g} .
$$

\footnotetext{
${ }^{5}$ Quaternions appear because $U(n, \mathbb{H})$ is the maximal compact subgroup of $S p(2 n, \mathbb{C})$
} 
Now

$$
\begin{gathered}
\operatorname{Tr}\left(A p_{q}\right)=\operatorname{Tr}\left(A q \bar{q}^{T}\right)+\operatorname{Tr}\left(A J \bar{q} q^{T} J\right)=\bar{q}^{T} A q+q^{T} J A J \bar{q} \\
=\bar{q}^{T} A q+\bar{q}^{T} J A^{T} J q=2 \bar{q}^{T} A q=-2 \omega(A J \bar{q}, q) .
\end{gathered}
$$

Thus,

$$
F_{m}^{\prime}(A)=\int_{|q|=1} \omega(B \bar{q}, q) \omega(C \bar{q}, q)^{m} d q
$$

where $B=x y^{T}-y x^{T}$ and $C=-2 A J$. Define $F_{m} \in S\left(\mathfrak{g l}_{2 n}(\mathbb{C})\right)$ by

$$
F_{m}(M):=\int_{|q|=1} \omega(M \bar{q}, q)^{m+1} d q, \quad M \in \mathfrak{g l}_{2 n}(\mathbb{C}) .
$$

Note that $\left.d F_{m}\right|_{C}(B)=(m+1) F_{m}^{\prime}(A)$.

We compute $F_{m}$ as in the previous case, by considering

$$
\int_{V} \omega(M \bar{q}, q)^{m+1} e^{-\zeta \bar{q}^{T} q} d q
$$

Now,

$$
\begin{aligned}
\sum_{m=-1}^{\infty} \frac{1}{(m+1) !} \int_{V} \omega(M \bar{q}, q)^{m+1} e^{-\zeta \bar{q}^{T} q} d q & =\int_{V} e^{-\bar{q}^{T}\left(\zeta-M^{T} J\right) q} d q \\
& =\pi^{2 n} \operatorname{det}\left(\zeta-M^{T} J\right)^{-1} \\
& =\pi^{2 n} \operatorname{det}(\zeta+J M)^{-1}
\end{aligned}
$$

Hence, $F_{m}(M)$ is equal to a constant times the coefficient of $\tau^{m+1}$ in the expansion of $\operatorname{det}(1+\tau J M)^{-1}$ as a power series in $\tau$. Differentiating $\operatorname{det}(1+\tau J M)^{-1}$ and evaluate at $B$, we get

$$
-\operatorname{Tr}\left(\tau J B(1+\tau J M)^{-1}\right) \operatorname{det}(1+\tau J M)^{-1} .
$$

Substituiting $B=x y^{T}-y x^{T}, M=C=-2 A J$, and replacing $\tau$ by $\tau / 2$, we get

$$
\begin{aligned}
& -\tau \operatorname{Tr}\left(J\left(x y^{T}-y x^{T}\right)\left(1-\tau A^{T}\right)^{-1}\right) \operatorname{det}(1-\tau A)^{-1} / 2 \\
& =\tau\left(\omega\left(x,(1-\tau A)^{-1} y\right)-\omega\left(y,(1-\tau A)^{-1} x\right)\right) \operatorname{det}(1-\tau A)^{-1} / 2 \\
& =\tau \omega\left(x,\left(1-\tau^{2} A^{2}\right)^{-1} y\right) \operatorname{det}(1-\tau A)^{-1} .
\end{aligned}
$$




\section{Representation theory of continuous Cherednik algebras.}

In this section we will consider the basics of representation theory of continuous Cherednik algebras $\mathrm{H}_{\kappa}(G)$, where $G \subset G L(\mathfrak{h}), V=\mathfrak{h} \oplus \mathfrak{h}^{*}$. For simplicity we will assume that $\mathfrak{h}$ is a faithful representation (so $t \in \mathbb{C}$ ).

By a representation of $\mathrm{H}_{\kappa}(G)$ we always mean a continuous representation, i.e., a locally finite representation of $G$ together with a compatible action of $V$ satisfying the main commutation relation.

5.1 Dunkl operators. Let $g \in G L(\mathfrak{h})$ be an element such that the operator $1-g$ has rank 1. Let $f \in \mathbb{C}[\mathfrak{h}]$. Then the function $f(u)-f(g u)$ is divisible by $\beta(u)$, where $\beta \in \operatorname{Im}\left(1-g^{-1 *}\right)$ is a basis element. Thus for any $y \in \mathfrak{h}$ we can define the regular function

$$
K_{f}(g, y, u):=\beta(y) \frac{f(u)-f(g u)}{\beta(u)}
$$

on $\left(S_{G L(\mathfrak{h})} \backslash\{1\}\right) \times \mathfrak{h} \times \mathfrak{h}$ (it is independent on the choice of $\beta$ ). Since $S_{G L(\mathfrak{h})}$ is normal, this function extends to $g=1$ (by zero). Hence $K_{f}$ may be regarded as a regular function on the scheme $\Psi \times \mathfrak{h} \times \mathfrak{h}\left(\right.$ as $\left.\Psi=S_{G L(\mathfrak{h})} \cap G\right)$.

Now let $c$ be a $G$-invariant distribution on $\Psi$. For any $y \in \mathfrak{h}$, define the Dunkl operator $D_{y}$ on $\mathbb{C}[\mathfrak{h}]$ by the formula

$$
\left(D_{y} f\right)(u)=t \partial_{y} f(u)+\left(c, K_{f}(?, y, u)\right) .
$$

(Here the last term means the evaluation of the distribution $c$ on the function $K_{f}(?, y, u)$ of $g$ for fixed $y, u$ ).

Theorem 5.1. Let $\kappa(x, y)=-t(x, y)-c(x,(1-g) y)$, and $\mathrm{H}_{\kappa}$ be the corresponding continuous Cherednik algebra. The assignment $x \rightarrow x$ for $x \in \mathfrak{h}^{*}, g \rightarrow g$ (action of $g$ on $\mathbb{C}[\mathfrak{h}]$ ), and $y \rightarrow D_{y}$ defines a representation of $\mathrm{H}_{\kappa}$ on $\mathbb{C}[\mathfrak{h}]$.

Proof. Let $\mathrm{H}_{\kappa}^{+}$be the subalgebra of $\mathrm{H}_{\kappa}$ generated by $\mathfrak{h}$ and $\mathcal{O}(G)^{*}$. Let $\mathbb{C}$ be the trivial representation of this algebra, where $y \in \mathfrak{h}$ acts by zero, and $g \in G$ by 1 . Let $M:=$ $\mathrm{H}_{\kappa} \otimes_{\mathrm{H}_{\kappa}^{+}} \mathbb{C}$. It follows from the PBW theorem for $\mathrm{H}_{\kappa}$ that $M=\mathbb{C}[\mathfrak{h}]$, where $x \in \mathfrak{h}^{*}$ acts by $x$ and $g \in G$ by $g$. It remains to compute the action of $y$. Let $x_{1}, \ldots, x_{r} \in \mathfrak{h}^{*}$. Then we have

$$
\begin{gathered}
y \circ x_{1} \ldots x_{r}=\left[y, x_{1} \ldots x_{r}\right] \cdot 1=\sum_{i} x_{1} \ldots x_{i-1}\left[y, x_{i}\right] x_{i+1} \ldots x_{r} \cdot 1= \\
\sum_{i}\left(t\left(y, x_{i}\right) x_{1} \ldots x_{i-1} x_{i+1} \ldots x_{r} \cdot 1+\left(c,\left(y, x_{i}-x_{i}^{g}\right) x_{1} \ldots x_{i-1} x_{i+1}^{g} \ldots x_{r}^{g} \cdot 1\right)=\right. \\
t \partial_{y}\left(x_{1} \ldots x_{r}\right)+\left(c, K_{x_{1} \ldots x_{r}}(?, y, u)\right) .
\end{gathered}
$$

(Here as usual $(c, f)$, for $f \in \mathcal{O}(G)$, is the evaluation of the distribution $c$ on the function $f)$. The theorem is proved. 
The representation defined above is called the Dunkl-Cherednik representation of the Cherednik algebra $\mathrm{H}_{\kappa}$. If $G$ is finite, it is the usual Dunkl-Cherednik representation of the rational Cherednik algebra.

Corollary 5.2. We have $\left[D_{y}, D_{y^{\prime}}\right]=0$ for any $y, y^{\prime} \in \mathfrak{h}$.

5.2 Category $\mathcal{O}$. Consider a continuous Cherednik algebra $\mathrm{H}_{\kappa}$, with $t=1$ and arbitrary $c$. Let $\operatorname{dim} \mathfrak{h}=d$. Like for usual Cherednik algebras, we can define the Euler element

$$
\mathbf{h}=c+\sum_{i} x_{i} y_{i}+\frac{d}{2}
$$

where $y_{i}$ is a basis of $\mathfrak{h}$ and $x_{i}$ the dual basis of $\mathfrak{h}^{*}$. This element commutes with $\mathcal{O}(G)^{*}$.

Proposition 5.3. We have $[\mathbf{h}, x]=x,[\mathbf{h}, y]=-y$ for $x \in \mathfrak{h}^{*}, y \in \mathfrak{h}$.

Proof. We have

$$
[\mathbf{h}, x]=[c, x]+\sum_{i} x_{i}\left[y_{i}, x\right]=[c, x]+x+(1-g) x \cdot c=x .
$$

The second identity is proved similarly.

Definition 5.4. Category $\mathcal{O}$ for $\mathrm{H}_{\kappa}$ consists of finitely generated $\mathrm{H}_{\kappa}$-modules which are locally finite with respect to $\mathbf{h}$, with real part of the spectrum of $\mathbf{h}$ bounded below.

The properties of category $\mathcal{O}$ are as usual; let us summarize some of them. The proofs are standard, see GGOR.

1. Any object of $\mathcal{O}$ is a finitely generated $\mathbb{C}[\mathfrak{h}]$-module.

2. For every irreducible finite dimensional $G$-module $Y$ we can define the Verma module $M(Y)=\mathrm{H}_{\kappa} \otimes_{\mathrm{H}_{\kappa}^{+}} Y$ (where $y \in \mathfrak{h}$ act in $Y$ by zero). In particular, $M(\mathbb{C})$ is the Dunkl-Cherednik representation. The module $M(Y)$ has an irreducible quotient $L(Y)$, and every irreducible object in $\mathcal{O}$ is isomorphic to $L(Y)$.

Remarks. 1. In the case of Hermitian symmetric pairs $\left(\mathrm{H}_{\kappa}=A(\mathbf{G}, \mathbf{K})\right)$, the modules $L(Y)$ (for appropriate $Y$ ) are the representations of "holomorphic discrete series".

2. For $c=0$, one has $M(Y)=L(Y)$, and category $\mathcal{O}$ is semisimple. This means that this is so for Weil generic $c$, i.e. outside a countable union of algebraic hypersurfaces. (An interesting problem is to find exactly for which $c$ this happens.)

3. For any module $M \in \mathcal{O}$, we can define the character $\chi_{M}=\operatorname{Tr}_{M}\left(g t^{\mathbf{h}}\right)$, which is a series in $t$ with coefficients in the representation ring of $G$. For example,

$$
\chi_{M(Y)}=\frac{t^{c_{Y}+d / 2}}{\left.\operatorname{det}\right|_{\mathfrak{h}^{*}}(1-g t)},
$$


where $c_{Y}$ is the eigenvalue of $c$ in $Y$. One of the main problems of representation theory of $\mathrm{H}_{\kappa}$ is to find the characters of $L(Y)$.

To keep this paper short, we will not develop representation theory of continuous Cherednik algebras systematically. Rather, to give the reader the flavor of this theory, we will study the question of reducibility of the Dunkl-Cherednik representation for $G=$ $G L(\mathfrak{h})$ and $G=O(\mathfrak{h}) .{ }^{6}$

\subsection{Representations of $\mathrm{H}_{\kappa}$ for $G=G L(\mathfrak{h})$.}

Proposition 5.5. (i) The Dunkl-Cherednik representation $M(\mathbb{C})$ has singular vectors in degree $N$ if and only if

$$
c_{S^{N} \mathfrak{h}^{*}}-c_{\mathbb{C}}=N .
$$

Thus $M(\mathbb{C})$ is irreducible iff equality (5.6) fails for all $N \geq 1$.

(ii) If $N$ is the smallest positive solution of (5.6) then $L(\mathbb{C})=\oplus_{j=0}^{N-1} S^{j} \mathfrak{h}^{*}$ is the quotient of $\mathbb{C}[\mathfrak{h}]$ by the ideal generated by polynomials of degree $N$.

Proof. (i) The action of $y \in \mathfrak{h}$ defines a homomorphism of $G$-modules $\mathfrak{h} \otimes S^{N} \mathfrak{h}^{*} \rightarrow S^{N-1} \mathfrak{h}^{*}$. There is only one such homomorphism up to scaling, so its vanishing is equivalent to one linear equation in $c$. If this equation is satisfied then there is a nontrivial homomorphism $M\left(S^{N} \mathfrak{h}^{*}\right) \rightarrow M(\mathbb{C})$ of degree $N$, which implies (5.6). Thus the linear equation in question is exactly (5.6), as desired.

(ii) is clear from the proof of (i).

5.4 Representations of $\mathrm{H}_{\kappa}$ for $G=O(\mathfrak{h})$. Let $G=O(\mathfrak{h}), \operatorname{dim}(\mathfrak{h})=d$. We fix an isomorphism $\mathfrak{h} \rightarrow \mathfrak{h}^{*}$ defined by the inner product on $\mathfrak{h}$. Let $s \in O(\mathfrak{h})$ be an orthogonal reflection, and $\operatorname{Ad}(G) s=S \backslash\{1\}$ be the conjugacy class of reflections.

In this case, it is sufficient to consider $c=k \Delta$, where $\Delta$ is the integral over the conjugacy class of reflections (=invariant projection to the trivial $G$-subrepresentation $\mathcal{O}(\operatorname{Ad}(G) s) \rightarrow \mathbb{C})$, and $k \in \mathbb{C}$. Thus we will denote the algebra $\mathrm{H}_{\kappa}$ with $t=1$ by $\mathrm{H}_{k}$.

The algebra $\mathrm{H}_{k}$ has an $s l(2)$ subalgebra, given by $F=\frac{1}{2} \sum x_{i}^{2}, E=-\frac{1}{2} \sum y_{i}^{2}, H=$ $-\frac{1}{2}\left(x_{i} y_{i}+y_{i} x_{i}\right)$ (it is easy to show that $H=-\mathbf{h}$ ).

The highest eigenvalue of $H$ on the Verma module $M(Y)$ is

$$
\lambda_{Y}(k)=-\frac{d}{2}-k \frac{\left.\operatorname{Tr}\right|_{Y}(s)}{\operatorname{dim}(Y)}
$$

This shows that in order to have a nontrivial map $M(Y) \rightarrow M\left(Y^{\prime}\right)$, the number $k$ must be rational. Thus, if $k$ is not rational, the category $\mathcal{O}=\mathcal{O}_{k}$ is semisimple, as $M(Y)=L(Y)$ for all $Y$.

\footnotetext{
${ }^{6}$ Note that for $G=G L_{d}$, the representation theory of $\mathrm{H}_{\kappa}$ is in some sense a "deformation" of representation theory of the Lie group $S U(d, 1)$ (with infinitely many parameters)
} 
Definition. The Shapovalov form $M(Y) \times M\left(Y^{*}\right) \rightarrow \mathbb{C}$ is the bilinear $G$-invariant form coinciding with the standard one on $Y \times Y^{*}$, and satisfying the equations $(y v, w)=$ $\left(v, y^{*} w\right),(v, y w)=\left(y^{*} v, w\right)$, where $y \in \mathfrak{h}$, and $y^{*} \in \mathfrak{h}^{*}$ is the element corresponding to $y$ under the identification $\mathfrak{h} \rightarrow \mathfrak{h}^{*}$.

It is easy to show that the Shapovalov form exists and is unique. Let $K(Y)$ be the kernel of the Shapovalov form, then $L(Y)=M(Y) / K(Y)$.

Let us study the kernel $K(Y)$. Any representation of $\mathrm{H}_{k}$ is in particular a representation of the dual pair $(G, s l(2))$. Moreover, for generic $k, M(Y)=\oplus_{n \geq 0} Q(n, Y) \otimes M_{\lambda_{Y}(k)-n}$, where $M_{\mu}$ is the highest weight Verma module over $s l(2)$ of highest weight $\mu$, and $Q(n, Y)$ is the finite dimensional space of highest weight vectors of degree $n$ under $\operatorname{sl}(2)$. From character considerations, it is easy to see that the representation $Q(n, Y)$ of $G$ is isomorphic to $\operatorname{Harm}(n) \otimes Y$, where $\operatorname{Harm}(n)$ is the space of harmonic polynomials of degree $n$.

Let us now study the structure of the Dunkl-Cherednik representation $M(\mathbb{C})$; i.e., $Y=\mathbb{C}, \lambda_{Y}(k)=: \lambda(k)=-d / 2-k$. Let $L_{m}$ denote the finite dimensional irreducible representation of $\operatorname{sl}(2)$ with highest weight $m\left(m \in \mathbb{Z}_{+}\right)$.

Proposition 5.7. If $k=-d / 2-m, m \in \mathbb{Z}_{+}$, then $L(\mathbb{C})=\oplus_{n=0}^{m} L_{m-n} \otimes \operatorname{Harm}(n)$. Otherwise $L(\mathbb{C})=M(\mathbb{C})$.

Remark. Let $d=2$, and $k=-1-m$. Then the representation $L(\mathbb{C})$ has dimension $k^{2}$ and is a limit of representations of dimension $k^{2}$ for finite dihedral groups $G$ found in Theorem 3.3.1 of [Ch] (first row of the table).

Proof. Since all representations $\operatorname{Harm}(n)$ are irreducible and non-isomorphic, for any $k$ we have $M(\mathbb{C})=\oplus_{n \geq 0} M(n) \otimes \operatorname{Harm}(n)$, where $M(n)$ is an $s l(2)$-module having the same character as $M_{\lambda(k)-n}$. But since $F=r^{2}:=\sum x_{i}^{2}$, the module $M(\mathbb{C})$ has no singular vectors under $F$. Hence $M(n)=M_{\lambda(k)-n}$, and thus $M(\mathbb{C})=\oplus_{n \geq 0} M_{\lambda(k)-n} \otimes \operatorname{Harm}(n)$.

Thus the Shapovalov form on $M(\mathbb{C})$ is the sum of the tensor product forms on $M_{\lambda(k)-n} \otimes \operatorname{Harm}(n)$ with coefficients. For nonnegative integer $\lambda(k)-n$, the Shapovalov form must vanish on $M_{-\lambda(k)+n-2} \subset M_{\lambda(k)-n}$. Furthermore, we claim that all $M_{\lambda(k)-n} \otimes \operatorname{Harm}(n)$ with $n>\lambda(k)$ belong to the kernel $K(Y)$. Indeed, $K(Y)$ is a $G$ invariant proper graded ideal in $\mathbb{C}[\mathfrak{h}]$. So its zero set is either the cone $r^{2}=0$ or the origin. In particular, $\left.r^{2 n}\right|_{L(Y)}=0$ for some $n$. This proves the claim.

Thus, the zero set of $K(Y)$ is the origin, and $L(Y)$ is identified with a $S L(2) \times O(d)$ subrepresentation of the finite dimensional representation $\oplus_{n=0}^{\lambda(k)} L_{\lambda(k)-n} \otimes \operatorname{Harm}(n)$.

It remains to show that this subrepresentation is in fact the entire representation. To do so, note that the component $L_{\lambda(k)}$ corresponding to $n=0$ must occur, as it contains the highest weight vector. This implies that $r^{2 p} \neq 0$ in $L(\mathbb{C})$ for $p=0, \ldots, \lambda(k)$. Now consider the component corresponding to some $n \leq \lambda(k)$. Consider its $s l(2)$-highest weight part, $\operatorname{Harm}(n)$. This is a representation of $O(d)$ defined over the real numbers, so it has a real basis $f_{i}$ orthonormal under the invariant inner product. Consider the function $F=\sum_{i} f_{i}^{2}$. 
This function is rotationally invariant and generically positive on the real subspace (as it is a sum of squares), so it is a nonzero multiple of $r^{2 n}$. This implies that the vector $\sum f_{i} \cdot f_{i}$, where the first factor $f_{i}$ belongs to $\mathrm{H}_{k}$ and the second one to $L(\mathbb{C})$, is nonzero. So at least one $f_{i} \in L(\mathbb{C})$ is nonzero, and the entire $n$-th summand must occur in $L(\mathbb{C})$.

The proposition is proved.

Remark. In fact, the proof of the proposition shows that any representation of $\mathrm{H}_{k}$ from the category $\mathcal{O}$ which does not have full support in $\mathfrak{h}$ is finite dimensional (as it is $s l(2)$-finite). So, the quotient of category of $\mathcal{O}$ by the category of finite dimensional representations maps injectively into $\operatorname{Rep} O(n-1)$ through the fiber functor at a generic point of $\mathfrak{h}$. This functor is somewhat analogous to the KZ functor from [GGOR.

\section{Case of wreath-products}

6.1 Wreath products. Many interesting examples of continuous symplectic reflection algebras may be obtained by means of wreath-product type construction. Specifically, let $\Gamma$ be a reductive subgroup of $S L_{2}$, and $G=S_{n} \ltimes \Gamma^{n} \subset S p_{2 n}$. When $\Gamma$ is finite (i.e., corresponds to a simply-laced affine Dynkin diagram), the corresponding symplectic reflection algebras were considered in [EG]. Thus here we will consider only the cases when $\Gamma$ is infinite. There are three such subgroups $\Gamma$ :

Example 6.1. $\Gamma=\mathbb{C}^{*}=G L_{1}$ (the maximal torus). In this case $S / G=\mathbb{C}^{*} \cup\{s\}$, where $s$ the conjugacy class of $s_{i j} \cdot{ }^{7}$

Example 6.2. $\Gamma=\widetilde{O}_{2}$ (the normalizer of the maximal torus). In this case, $S / G=$ $\mathbb{C}^{*} \cup\{s, \sigma\}$, where $\sigma$ is a non-diagonal element of $\widetilde{O}_{2}$.

Example 6.3. $\Gamma=S L_{2}$. In this case, $S / G=\mathbb{C}^{*} \cup\{s\}$.

These correspond to the infinite affine Dynkin diagrams $A_{\infty}, D_{\infty}, A_{\infty} / 2$ depicted in Figure 1 by the standard rule of McKay's correspondence (see below).

Proposition 6.4. Let $G$ be as in example 6.16.2, or 6.3. Then the most general continuous symplectic reflection algebra for $G$ is obtained by taking $c \in \mathrm{C}(S)$.

Proof. In Example 6.1, it is easy to show that $\Psi$ is reduced (i.e., $\Psi=S$ ), and thus the statement follows from Corollary 3.11

In examples 6.26.3, we have $\Sigma=\Sigma_{0} \cup A d(G) s, S=S_{0} \cup A d(G) s$, where $\Sigma_{0}$ and $S_{0}$ are the intersections of $\Sigma$ and $S$ with $G_{0}:=\Gamma^{n}$. Also, one has $\mathbb{C}\left[G_{0}\right]^{G_{0}}=\mathbb{C}\left[T_{1}, \ldots, T_{n}\right]$, where the functions $T_{i}$ are defined by the formula $T_{i}\left(\gamma_{1}, \ldots, \gamma_{n}\right)=\operatorname{Tr}\left(\gamma_{i}-1\right)$. The defining equation of $\Sigma$ introduces the relations $T_{i} T_{j}=0, i \neq j$. This implies that $\Sigma / G=S / G$, and the statement follows.

\footnotetext{
${ }^{7}$ In all three examples, the class $s$ is absent if $n=1$.
} 


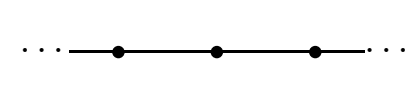

$A_{\infty}$

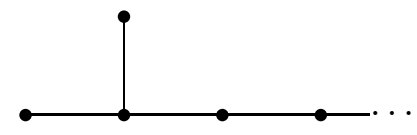

$D_{\infty}$

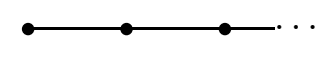

$A_{\infty / 2}$

Figure 1: Graphs associated to $G L_{1}, \widetilde{O}_{2}$, and $S L_{2}$.

We see that in all three examples, for $n>1$ one has $\mathrm{C}(S)=\mathrm{C}\left(S_{0}\right) \oplus \mathbb{C} \Delta$, where $\Delta$ is the integral over $A d(G) s$. Moreover $\mathrm{C}\left(S_{0}\right)$ is naturally isomorphic to $\mathrm{C}(\Gamma)$ (as $\Gamma$ is $S$ for $n=1)$; we will identify these spaces using this isomorphism.

6.2 Representations of wreath product algebras. The theory of wreath product algebras for infinite groups $\Gamma$ is rather similar to the theory in the finite case. As an example let us work out finite dimensional representatations of $\mathrm{H}_{\kappa}$ which are irreducible as $G$-modules. For finite groups $\Gamma$, this was done in $[\mathbf{M}]$. Since the proofs in the infinite case are the same as in the finite one, we will omit them.

As before, we will denote by $\Gamma$ one of the groups $G L_{1}, \widetilde{O}_{2}$ or $S L_{2}$, and let $G:=S_{n} \ltimes \Gamma^{n}$. We shall write $L$ for the 2-dimensional tautological representation of $\Gamma ; V=L^{\oplus n}$.

Let $\left\{Y_{i}\right\}_{i \in I}$ be the set of finite dimensional irreducible representations (up to isomorphism) of $\Gamma$. We associate a graph to $\Gamma$ in the following way: the set of vertices of the graph is $I$, and the number of edges joining $i, j \in I$ is the multiplicity of $Y_{i}$ in $L \otimes Y_{j}$. See Figure 1 , where $A_{\infty}, D_{\infty}$, and $A_{\infty / 2}$ are the graphs associated, respectively, to $G L_{1}, \widetilde{O}_{2}$, and $S L_{2}$.

In the theorem below, we shall use the following notations. Let $\underline{n}=\left(n_{i}\right)_{i \in I}$, where $n_{i} \in \mathbb{Z}_{\geq 0}$, and $\sum_{i \in I} n_{i}=n$. Let $I(\underline{n}):=\left\{i \in I \mid n_{i}>0\right\}$. Let $Y:=\bigotimes_{i \in I(\underline{n})} Y_{i}^{\otimes n_{i}}$. For each $i \in I(\underline{n})$, we shall regard $S_{n_{i}}$ as the subgroup of $S_{n}$ which permutes the factors in $Y_{i}^{\otimes n_{i}}$ in $Y$. Let $S_{\underline{n}}:=\prod_{i \in I(\underline{n})} S_{n_{i}} \subset S_{n}$. Let $W_{i}$ be an irreducible representation of $S_{n_{i}}$, and let $W:=\bigotimes_{i \in I(\underline{n})} W_{i}$. Thus, the group $S_{\underline{n}} \ltimes \Gamma^{n}$ acts on $W \otimes Y$ (where $\Gamma^{n}$ acts trivially on $W$ ). Denote by $W \otimes Y \uparrow$ the (irreducible) representation of $G$ induced from the representation $W \otimes Y$ of its subgroup $S_{\underline{n}} \ltimes \Gamma^{n}$.

We also let

$$
\kappa(x, y)=\omega(x, y) \delta_{1}+\omega((1-g) x,(1-g) y)(c+k \Delta),
$$

where $c \in \mathrm{C}(\Gamma)$. We know that this gives rise to the most general continuous symplectic reflection algebra (with $t=1$ ).

Theorem 6.5. (i) Assume $k \neq 0$. The representation $W \otimes Y \uparrow$ of $G$ extends to a representation of $\mathrm{H}_{\kappa}(G)$ where elements of $V$ act by 0 , if and only if the following conditions are satisfied: 
(1) For all $i \in I(\underline{n})$, the irreducible representation $W_{i}$ of $S_{n_{i}}$ has rectangular Young diagram, of size $a_{i} \times b_{i}$.

(2) For all $i, j \in I(\underline{n})$ (where $i \neq j$ ), the vertices $i$ and $j$ in the graph associated to $\Gamma$ are not adjacent.

(3) For each $i \in I(\underline{n})$, one has

$$
\operatorname{dim} Y_{i}+2 k\left(b_{i}-a_{i}\right)+\left(c, \chi_{Y_{i}}(g) \operatorname{det}\left(1-\left.g\right|_{L}\right)\right)=0,
$$

where $\chi_{Y_{i}}$ is the character of $Y_{i}$.

(ii) If $E$ is a finite dimensional representation of $\mathrm{H}_{\kappa}(G)$ which is irreducible as a $G$ module, then $V$ acts by zero in $E$; in particular, $E$ is as specified in (i).

The proof of Theorem 6.5] is similar to the proof of [M], Theorem 3.1, Theorem 4.1].

\section{References}

[BG] A. Braverman, D. Gaitsgory, Poincaré-Birkhoff-Witt theorem for quadratic algebras of Koszul type, J. Algebra 181 (1996), no.2, 315-328, hep-th/9411113.

[BGS] A. Beilinson, V. Ginzburg, W. Soergel, Koszul duality patterns in Representation Theory, J. Amer. Math. Soc. 9 (1996), 473-527.

[Ch] T. Chmutova, Representations of the rational Cherednik algebras of dihedral type, math.RT/0405383

[DG] M. Demazure, P. Gabriel, Introduction to algebraic geometry and algebraic groups, North-Holland Math. Studies 39, North-Holland Publishing Co., Amsterdam-New York, 1980.

[Dr1] V. Drinfeld: On quadratic commutation relations in the quasiclassical case, Mathematical physics, functional analysis (Russian), 25-34, 143, "Naukova Dumka", Kiev, 1986. Selecta Math. Sovietica. 11 (1992), 317-326.

[Dr2] Drinfeld, V. G. Degenerate affine Hecke algebras and Yangians. (Russian) Funktsional. Anal. i Prilozhen. 20 (1986), no. 1, 69-70.

[EG] P. Etingof, V. Ginzburg, Symplectic reflection algebras, Calogero-Moser space, and deformed Harish-Chandra homomorphism, Invent. Math. 147 (2002), 243-348, math. AG/0011114.

[GGOR] Ginzburg, V., Guay, N., Opdam, E., Rouquier, R., On the category $\mathcal{O}$ for rational Cherednik algebras. Invent. Math. 154 (2003), no. 3, 617-651.

[HE] M. Hochster, J. Eagon, Cohen-Macaulay rings, invariant theory, and the generic perfection of determinantal loci, Amer. J. Math. 93 (1971), 1020-1058.

[Kh] A. Khare, Category $O$ over a deformation of the symplectic oscillator algebra, J. Pure and Applied Algebra 195 (2005), no. 2, 131-166, math.RT/0309251.

$[\mathrm{Ku}] \quad \mathrm{R}$. Kutz, Cohen-Macaulay rings and ideal theory in rings of invariants of algebraic groups, Trans. Amer. Math. Soc. 194 (1974), 115-129.

[M] S. Montarani, On some finite dimensional representations of symplectic reflection algebras associated to wreath products, preprint, math.RT/0411286.

[RS] Ram, A., Shepler, A. V., Classification of graded Hecke algebras for complex reflection groups, Comment. Math. Helv. 78 (2003), no. 2, 308-334. 
Department of Mathematics, Massachusetts Institute of Technology, Cambridge, MA 02139, USA etingof@math.mit.edu

Department of Mathematics, Massachusetts Institute of Technology, Cambridge, MA 02139, USA

wlgan@math.mit.edu

Department of Mathematics, University of Chicago, Chicago, IL 60637, USA

ginzburg@math.uchicago.edu 